\title{
Proceedings of the British Cardiac Society
}

THE AUTUMN MEETING of the British Cardiac Society was held at the Wembley Conference Centre, London, on Monday and Tuesday, 3 and 4 December 1984. The President, MICHAEL OLIVER, took the Chair during private business. At the scientific sessions the Chair was taken by HUGH BENTALL.

\section{Abstracts of papers}

\section{Homograft aortic root replacement for complicated prosthetic valve endocarditis}

T Crake, D N Ross, $M$ Yacoub, $R$ Donaldson

National Heart Hospital, London

Thirteen adult patients (mean age 41 years) with prosthetic valve endocarditis or uncontrolled sepsis and aortic root abscesses underwent homograft aortic root replacement with reimplantation of the coronary arteries. There were two perioperative deaths. The endocarditis was cured in all 11 survivors, who have been followed up from 1 to 132 months (mean 33 months). Two of these patients are having symptoms due to homograft valve malfunction; excellent clinical and haemodynamic results have been obtained in the other nine patients.

Homograft aortic root replacement excludes the root abscesses and the weakened infected aortic annulus from the high systemic pressure and permits suturing of the homograft to healthy myocardium. It provides an alternative technique in the management of selected patients with active prosthetic infection and aortic ring destruction by sepsis.

Adult heart valve dimensions and their surgical implications

S Westaby, R B Karp, E H Blackstone

Royal Postgraduate Medical School, London, and University of Alabama, Birmingham, USA

After valve replacement, some patients fail to improve in functional status because their prosthesis is restrictive. We believe that for adults, body habitus relates poorly to valve size and that Lev's data relating body surface area to valve size in childhood cannot be extrapolated into adult life. We therefore sought guidelines for valve replacement by measuring valve circumference, then calculated circularised orifice areas in 160 postmortem hearts from adults with and without congestive cardiac failure. Multivariate analysis was used to relate orifice area to age, sex, height, body surface area, and congestive cardiac failure. We found only sex and congestive cardiac failure to have statistically significant relations to valve area, correlation coefficients showing body surface area and other variables to be poorly related. We can therefore state a mean circularised orifice area for each adult cardiac valve and aim to insert an appropriate prosthesis. Male patients have larger valves, and atrioventricular valves are enlarged in congestive cardiac failure. Comparison of mean valve area with the effective orifice area of current prostheses shows, however, the majority (particularly mechanical valves) to be restrictive at rest, especially in the mitral and tricuspid positions. Improved prosthetic design, valve repair wherever possible, and annulus enlargement procedures are required to eliminate this problem.

\section{Early and medium term results of cardiac transplantation with minimum or no oral steroids}

M Yacoub, P Alivizatos, Rosemary Radley-Smith, K Khagani, A Mitchell

Harefield Hospital, Harefield, Middlesex

Between February 1980 and August 1984, 124 patients underwent cardiac transplantation at Harefield Hospital with an overall one year survival of $72 \%$. Early in the experience oral steroids were identified as a major cause of morbidity and mortality, particularly in very young and elderly patients. Since September 1982 the immunosupressive protocol consisted of cyclosporin, azathioprine, and antithymocyte globulin with no or minimum 5-15 mg oral steroids/ day. During this period 85 patients underwent cardiac transplantation (65 orthotopic and 20 heterotopic). Their ages varied from 16 to 59 (mean 44.7) years. The original disease was ischaemic in 47 , congestive cardiomyopathy in 29 , valvular in six, and others in three. There were 15 deaths $(17.6 \%)$ all within the first three months, giving an actuarial one year survival of $82 \%$. Four patients (5\%) developed relatively serious infections, which were successfully treated 
Eight patients ( $9 \%$ ) required treatment for systemic hypertension and three (3.5\%) developed lymphoproliferative disease which regressed on reducing immunosupression in two and required excision in one. Twenty five patients, investigated by coronary angiography one year postoperatively, showed no localised or diffuse narrowing (chronic rejection). Graft function has been good in all survivors.

It is concluded that cardiac transplantation can be performed with minimum, or no, oral steroids and that this might help to liberalise the age limits for acceptance.

\section{Development of the clinical heart-lung transplant programme}

J Wallwork, R Cory-Pearce, T A H English

Papworth Hospital, Papworth Everard, Cambridgeshire

Since November 1982 progress has been made towards the development of clinical heart lung transplantation at Papworth Hospital. Between November 1982 and August 1984, there have been 59 referrals for heart-lung transplantation, 42 with primary lung disorders and 17 with primary cardiac defects. Many early referrals did not meet our criteria, primarily because of age or medical unsuitability. Fourteen patients have been assessed for transplantation and six await assessment; nine have been accepted as heartlung recipients. Two patients with primary pulmonary hypertension on prostacycline therapy have been provisionally accepted. Of the nine patients, three have died waiting. Four patients are currently awaiting transplantation, the major limiting factor being the availability of suitable donor organs. Size compatibility, normal lung function, and clear chest $x$ ray appearances are essential.

Between April 1984 and September 1984, two patients have been successfully transplanted, one with fibrosing alveolitis and one with Eisenmenger's syndrome secondary to an atrial septal defect. The patients are alive and well at six months and one month following transplantation with excellent cardiopulmonary function, $\mathrm{PO}_{2}>11 \mathrm{kPa}$ on air. We have introduced a new method of preserving donor lungs prior to transplantation, the preservation solution being colloid rather than crystalloid based. This has in our initial experience produced excellent early postoperative pulmonary function. Immunosuppression consisted of cyclosporin A, low dose steroids, and a short course of equine antithymocyte globulin. Imuran has not been used. Combined heart and lung transplantation is a viable clinical procedure for selected young patients with lethal and disabling pulmonary or cardiopulmonary disease.

Preoperative predictability of the late clinical outcome after CABG in patients with severe ventricular dysfunction

\section{P Sergeant, L Wouters, W Flameng, L Dekeyser, R Suy \\ University Hospital, Leuven, Belgium, and Janssen Pharma NV, Beerse, Belgium}

The predictability, based on preoperative factors, of the late clinical outcome after pure coronary artery bypass grafting is evaluated in 60 patients with a severe ventricular dysfunction (mean ejection fraction (EF) $31.8 \%$ and mean end diastolic pressure $17.6 \mathrm{~mm}$ $\mathrm{Hg}$.

Actuarial total survival, cardiac survival, and eventfree curves, angina, and NYHA classification at 12 and 18 months postoperatively, made for 15 preoperative variables, define the late clinical result. The cardiac operative mortality was $0 \%$ for the $35-45 \% \mathrm{EF}$ subgroup, $0 \%$ for the $25-34 \%$ EF subgroup, and 20\% for the under $24 \% \mathrm{EF}$ subgroup. The 24 months actuarial cardiac survival was $93 \%$ for the patients with a $35-45 \% \mathrm{EF}, 93 \%$ for the patients with a $25-$ $34 \% \mathrm{EF}$, and $43 \%$ for those with an EF under $24 \%$.

A mathematical model was constructed and the mortality predictability at 18 months was analysed by stepwise logistic regression. The best prediction was obtained using the ejection fraction and the preoperative NYHA functional class with $91.7 \%$ correct predictions, a sensitivity of $72.7 \%$, and a specificity of $95.9 \%$. The rank correlation between the predicted probability and the response was $\mathbf{0 . 9 2 2}$. Other variables did not increase the accuracy.

Probability $=\frac{\mathrm{e}^{-1.63-0.28 \times \mathrm{EF}+2.95 \times \mathrm{NYHA}}}{1+\mathrm{e}^{-1.63-0.28 \times \mathrm{EF}+2.95 \times \mathrm{NYHA}}}$

This formula can help to define the patients with poor ejection fraction but very good clinical outcome and those in whom the high operative risk cumulates with poor late survival. 
Myocardial revascularisation during acute infarction

P Sergeant, W Flameng, J Vanhaecke, L Dekeyser, R Suy

University Hospital, Leuven, Belgium

Thirty two patients were operated on during the acute phase of myocardial infarction. Their mean age was $53 \cdot 7(6 \cdot 8)$ years (range $36-69$ ). Of the patients (26 male and six female), $37 \%$ had previously suffered from another myocardial infarction. All patients were accepted for surgery whatever their clinical condition; $50 \%$ were in carcinogenic shock or receiving cardiac resuscitation. Myocardial revascularisation to the threatened area was finished in 18 patients less than 150 minutes after the onset of ischaemia, with a mean of $183.5(88.5)$ minutes. A mean of 2.3 distal anastomoses were constructed per patient. The operative mortality was $9.3 \%$. The operative survivors were followed for 15.5(8.3) months; all are alive at the end of follow up and $95 \cdot 7(4 \cdot 1) \%$ stayed angina free.

Postoperative radionuclide assessment, more than two months after surgery, showed significantly smaller thallium defects and better ejection fractions than controls $(n=20)$, who received conventional treatment for acute myocardial infarction, when surgery was finished within two hours. When revascularisation was completed more than four hours after the onset of symptoms, the scintigrams were not different from the controls. Electromicroscopical studies of epicardial and endocardial biopsies from the centre of the area at risk correlate with the radionuclide findings.

Coronary surgery can be performed in the acute phase of the myocardial infarction but should be limited to the early revascularisations if the damage to the cardiac function wants to be preserved.

Inhibition of implanted pacemakers by domestic electrical appliances

\author{
A K Agarwal, V J Redding \\ Groby Road Hospital (Regional Cardiac Centre), \\ Leicester
}

Unipolar ventricular inhibited demand cardiac pacing is the usual mode of treatment for symptomatic bradyarrythmias. Demand (VVI) pacemakers are susceptible to electromagnetic interference which may cause return of symptoms depending upon the dura- tion of induced inhibition and the patient's underlying spontaneous rhythm. Various methods have been tried to avoid external electromagnetic interference by metal housing of the unit and by modification of the pulse and amplitude discriminating sensing circuit. Because of the increasing use of electrical domestic appliances, the sources of electromagnetic interference are growing rapidly. It is important to have a working knowledge of the behaviour of modern pacemakers in the presence of such potential hazards.

Fifty four patients with implanted pacemakers of different manufacturers were tested against common domestic mains electrical appliances operating in close proximity to the unit at variable distances: shaver, hairdryer, food processor, domestic drill, hedge clipper, vacuum cleaner, and microwave oven. Significant pacemaker inhibition was induced in 37 patients by equipment operating at less than $22.5 \mathrm{~cm}$ proximity. The domestic drill (91\%) and the hedge clipper (41\%) were the main inhibitors. The shaver also affected a small number (7) of the patients.

This preliminary study demonstrates that some domestic electrical appliances can seriously interfere with pacemaker function. Operation of the equipment tested appears, however, to be safe beyond a distance of $30 \mathrm{~cm}$.

Left ventricular dynamics during exercise in physiological and non-physiological pacing modes using gated radionuclide angiography

W C Brownlee, D L Hastings

Departments of Cardiology and Medical Physics, Manchester Royal Infirmary, Manchester

Left ventricular dynamics have been compared during physiological ventricular fixed rate and externally triggered rate responsive ventricular pacing. Measurements of ventricular function were made using equilibrium gated radionuclide angiography at rest and during steady state exercise. Fifteen patients in complete heart block treated with programmable VAT or DDD pacemakers were studied first in the VAT mode and 14 days later in the VOO mode. In five of these patients an additional mode, externally triggered rate responsive ventricular pacing without atrioventricular synchrony, was employed. Indices of left ventricular volumes, cardiac output, and ejection fraction were derived. Comparing VAT and VOO modes two groups of patients could be identified according to the response of stroke volume on exercise in the VAT mode. Those able to increase stroke vol- 
ume on exercise (8) had a larger rise in cardiac output $(p<0.02)$, which was achieved by rate responsiveness and an increase in end diastolic volume. Comparison between the VAT and externally triggered ventricular pacing modes showed similar cardiac outputs at the same ventricular rates on exercise, but with atrioventricular synchrony the end diastolic volume was larger $(\mathrm{p}<0.01)$.

It is concluded that $(a)$ provided stroke volume can increase, cardiac output is improved by physiological pacing and $(b)$ atrioventricular synchronous pacing causes a higher end diastolic volume than ventricular pacing at the same rate.

\section{Digital analysis of atrial electrograms: a means of preventing pacemaker mediated re-entry tachycardia?}

D W Davies, R J Wainwright, M Tooley, A J Camm St Bartholomew's Hospital, London, and the Brook General Hospital, London

The inability of current pacemaker sensing circuits to distinguish anterograde from retrograde atrial depolarisation contributes to the genesis of pacemaker mediated re-entry tachycardia (PMRT). Slew rate analysis of digital atrial signals may overcome this problem.

Using temporary bipolar electrodes, electrograms were recorded simultaneously from three right atrial sites (appendage, high and low lateral) during sinus rhythm and right ventricular pacing in 10 patients with intact retrograde (V-A) conduction. The unprocessed electrograms were analysed for amplitude, slew rate, and signal polarity. These signals were then converted to digital form and further analysed by a slope detection program which displays the original electrogram as a sequence of positive and negative turning points with amplitudes proportional to the original slew rates.

Retrograde atrial signals fulfilled current pacemaker sensing criteria in all patients confirming the potential for PMRT. Significant overall differences between anterograde and retrograde signals were only seen in the slew rates at appendage and low lateral sites: $1 \cdot 1(0.5)$ (mean (SD)) vs $0.7(0.4)$ and $1.0(0.4)$ vs $0.7(0.2) \mathrm{V} / \mathrm{s}$ respectively ( $\mathrm{p}<0.05$ for both). Subtle visible differences between the morphology of the analogue anterograde and retrograde electrograms were observed in all patients which would not be detected by simple pacemaker sensing circuitry.
Nevertheless, analysis of the digital signal enhanced these differences to the point where anterograde and retrograde depolarisations could be recognised automatically in all patients from 1 or more atrial sites. Digital analysis of atrial signals has successfully distinguished anterograde from retrograde depolarisation and has important future applications in the pacemaker management of iatrogenic and spontaneous tachycardia.

Comparison of burst overdrive, self-searching, and adaptive table scanning programmes in antitachycardia pacing

\section{P Holt, J C P Crick, E Sowton Department of Cardiology, Guy's Hospital, London}

Seven patients with the Siemens Elema "Tachylog" generator implanted for the management of their paroxysmal tachycardias were studied to assess the safety and efficacy of three antitachycardia programmes. The modes studied were burst overdrive (BOD), self-search (SS), and adaptive table scanning (ATS). There were four men and three women aged 20-62 years. Six had Wolff-Parkinson-White (WPW) syndrome and one had ventricular tachycardia (VT). Three patients with WPW and one with VT had pacing wires to the right ventricle, the remainder had right atrial pacing electrodes.

All patients were studied acutely lying, standing, and post exercise in all three modes, and the appropriate number and interval of stimuli chosen for the long term programmes. The generator remained in one programme for one month, was interrogated, and the memory read, then reprogrammed to a different antitachycardia mode. BOD was unsuitable for long term use in four patients, producing atrial fibrillation in one and VT in three. In this group SS and ATS were safe and equally effective (mean number of "searches"/attack 6.8 and 6.2 respectively). In the three patients in whom BOD, SS, and ATS could all be safely used BOD proved most efficient, mean number of attempts-tachycardia $=3.6$ (cf 11.3 for SS and $12 \cdot 1$ for ATS).

Thus careful study and a versatile multiprogrammable generator are neccessary to provide the safest long term antitachycardia programme. Burst overdrive was suitable for only $42 \%$ of our patients, but when safe was especially valuable in those with narrow termination windows on exercise. 
Continuous measurement of ventricular refractory period and the prediction of imminent loss of capture from the ventricular paced evoked response

\author{
E J Perrins, L M Shapiro, E Rowland, R M \\ Donaldson, A F Rickards \\ National Heart Hospital, London
}

An external pacing system has been designed, which is capable of high fidelity recording of the unipolar evoked electrogram within $5 \mathrm{~ms}$ of an applied pacing stimulus. This allows accurate registration of the evoked QRS complex, ST segment, and T wave. There is a marked similarity between the time courses of the paced evoked response and the monophasic action potential recorded from the same site. It should be possible to predict the ventricular refractory period from the evoked stimulus to peak $T$ wave interval (St-T). The ventricular paced evoked response was recorded in five patients during programmed ventricular stimulation. The single ventricular extrastimulus technique was used to determine ventricular refractory period at basic paced cycle lengths of 280 $800 \mathrm{~ms}$. The St-T interval measured at each cycle length was then compared with the ventricular refractory period (Vref) at that pacing rate. A linear relation was observed (Vref $=0.94 \times \mathrm{St}-\mathrm{T}-22.1 \mathrm{~ms} ; \mathrm{r}=0.98$, $\mathrm{p}<0.001$ ) which was highly significant. In addition marked widening and fractionation of the evoked QRS complex was detected when the premature stimulus was delivered within $10-20 \mathrm{~ms}$ of the ventricular refractory period.

Similar changes in the evoked QRS complex were also seen with pacing energies less than 2-3 times diastolic threshold in six anaesthetised and thoracotomised dogs. The evoked QRS widened by a mean of $62 \mathrm{~ms}$ prior to loss of capture. These preliminary results suggest that prediction of both ventricular refractory period and imminent loss of capture is possible using this device and may find application in future antitachycardia pacemakers.

\section{Blood pressure and symptoms during pacing}

R I Jones, P M M Cashman, R S Horning, L Bassein, E B Raftery

Department of Cardiology, Northwick Park Hospital and Clinical Research Centre, Harrow, Middlesex

Patients with functioning pacemakers frequently have symptoms of presyncope. We have performed ambulatory monitoring of blood pressure in 10 patients (mean age 67 years) in sinus rhythm origi- nally paced (VVI) for the sick sinus syndrome. They were not taking any cardiovascular medication, and there was no evidence of pacemaker malfunction. Six were asymptomatic and four had symptoms of presyncope. Ambulatory blood pressure recordings were performed using a modification of the "Oxford" system which incorporated a pacing spike detector. From each tape episodes containing at least 20 beats of sinus rhythm followed by 20 beats of paced rhythm were selected. A total of 101 such episodes were analysed. Within each episode zero was defined as the onset of pacing and beats were numbered consecutively in a positive direction (after onset) and negatively (count down to onset). Eleven beat regression analysis of blood pressure on beat number was then performed. The number of quadratic components present at each beat number was then calculated and a $\chi^{2}$ test performed for systematic deviations between expected and observed distributions. All showed a significant $(p<0.01-p<0.001)$ fall in blood pressure with the onset of pacing. In the asymptomatic patients blood pressure recovered within five beats, but in the symptomatic group this recovery was delayed by up to 15 beats. Two symptomatic patients underwent a change of pacemaker to a DVI device. This resulted in the disappearance of symptoms and abolition of the fall in blood pressure. These data suggest that the onset of VVI pacing induces a fall in blood pressure in most patients, but symptoms only appear if baroreflex compensation is impaired. These haemodynamic consequences may be abolished by dual chamber pacing suggesting that baroreflex function should be tested before deciding which pacing mode to use.

Surgical correction of total anomalous pulmonary venous connexion: influence of cross sectional echocardiography and perioperative therapeutic optimisation

C Lincoln, C Mercanti, M Rigby, E Shinebourne, M Joseph, G Miller

Brompton Hospital, London

Sixty six patients have been surgically treated for correction of total anomalous pulmonary venous connexion (TAPVC) from 1973 to $1984 ; 38$ were male and 25 female. The median age at operation was 77 days and the median weight $3.8 \mathrm{~kg}$. The anatomic type encountered was infracardiac (IC) in 13, cardiac (C) in 27, supracardiac (SC) in 24, and mixed (MC) in 2. The diagnosis was established by catheterisation and angiography in 47 patients, in three by clinical examination, and in the last consecutive 16 by cross sec- 
tional echocardiography alone. Pulmonary hypertension (PHT) was present in $26(41 \%)$ of those patients catheterised preoperatively. Pulmonary vascular resistance (PVR) averaged $8.1 \mathrm{u} \mathrm{m}^{2}$, and in those with IC $>10 \mathrm{u} \mathrm{m}^{2}$. Conventional (CPB) bypass with profound to moderate hypothermia was used in 14 patients, and preliminary surface cooling then core cooling to $15^{\circ} \mathrm{C}$ with circulatory arrest in 52. Six patients developed one or more pulmonary hypertensive crises during the postoperative period, in four diagnosed by direct pulmonary artery pressure (PAP) measurement and in two on clinical examination. Late reoperation was necessåry in six (11\%) patients. Multivariant analysis of risk factors for $\mathbf{3 0}$ days survival with regard to sex, year of operation, days in hospital before operation, age, height, weight, body surface area, $\mathrm{pH}$ on admission, PAP, PVR, PHT crises, mode of investigation, type of CPB, and type of anatomy showed that risk of early death was increased in respect to PHT crises $(p<0.06)$, year of operation $(p<0.02)$, and sex of patient $(p<0.02)$ but decreased with echo diagnosis alone $(p<0.05)$. The 30 day hospital mortality from 1975 to the present time is $11 \%$. The late mortality is represented by six (9.5\%) patients. Actuarial survival of IC patients is $69 \%$ at 72 months, but the others had $88 \%$ survival at 80 months.

Continuous wave Doppler echocardiography in 120 infants and children with congenital heart disease
A B Houston, I A Simpson, C Sheldon, W B Doig, E N Coleman
Royal Hospital For Sick Children, Glasgow

Continuous wave Doppler echocardiography has been used to study 120 infants and children (7 days to 15 years) with congenital heart disease undergoing cardiac catheterisation or cardiac surgery without catheterisation. The provisional diagnosis was made with clinical examination and cross sectional echocardiography and the Doppler study directed to sites where a pressure gradient was suspected. The maximum velocity of blood flow was measured and the gradient calculated from the modified Bernoulli formula. An accurate estimate $(r=0.94)$ of the gradient was obtained in those with stenotic lesions including pulmonary valve stenosis (26), pulmonary valve and infundibular stenosis (16), aortic stenosis (10), pulmonary stenosis with transposition (4), and pulmonary artery banding (5). A satisfactory estimate of the pulmonary artery pressure in 17 patients with a ventricular septal defect was obtained by subtracting the estimated pressure difference between the ventricles from the systolic blood pressure. In 14 of 15 patients with coarctation of the aorta demonstration of increased velocity in the distal aortic arch confirmed evidence of the site of coarctation; in another failure to demonstrate this suggested complete obstruction. Doppler echocardiography provided information not obtained at catheterisation in six and which was important in undertaking surgery without catheterisation in two.

Continuous wave Doppler echocardiography has been shown to make an accurate assessment of pressure gradients between ventricles, over stenosed ventricular outlets, and across arterial obstructions and is thus an important non-invasive technique in the management of a wide variety of cardiovascular defects.

Clinical value of continuous wave Doppler in adults with aortic stenosis

I A Simpson, A B Houston, C D Sheldon, I Hutton, T D V Lawrie

Departments of Cardiology, Royal Infirmary and Yorkhill Hospital, Glasgow

Thirty five adults with clinical evidence of aortic stenosis (age range 15 to 71 years) were evaluated using continuous wave Doppler in addition to noninvasive assessment with clinical examination, ECG, chest $x$ ray, and cross sectional echocardiography. Doppler examination was performed using a Vingmed Alfred continuous wave Doppler velocimeter in conjunction with spectrum analysis, using a $2 \mathrm{MHz}$ transducer. Doppler gradients were calculated using the modified Bernoulli equation. Peak to peak pressure gradients were obtained independently at cardiac catheterisation in all patients, and simultaneous Doppler examinations performed in 25 . There was a good correlation between the two methods $(r=0.92)$, in particular when simultaneous Doppler recordings were obtained $(r=0.98)$. When the complete non-invasive assessment of these patients was evaluated, all those with a surgically significant stenosis were correctly identified. In particular, overestimation was not found in any patient with a less than significant obstruction.

In conclusion, therefore, continuous wave Doppler provides an accurate assessment of the degree of aortic stenosis, and in conjunction with other non-invasive techniques obviates the need for catheterisation in these patients. 
Assessment of stroke volume and cardiac output by Doppler ultrasound alone

\author{
J M Rawles, M K Daniel, Neva E Haites, Fiona M \\ McLennan, D H R Mowat \\ Department of Medicine, University of Aberdeen, \\ Aberdeen
}

It has been shown that stroke volume may be calculated from the product of aortic cross sectional area and the systolic velocity integral of aortic blood flow. On theoretical grounds, the systolic velocity integral (stroke distance) would therefore be expected to behave as the stroke volume index, and this was tested in healthy subjects and seven groups of patients. The normal range of aortic blood velocity was established in 140 healthy adults using a non-invasive Doppler ultrasound technique. Integration of the area under the velocity time curve for each beat gives stroke distance, and its product with heart rate gives minute distance. We have shown that stroke distance is independent of sex, body surface area, and blood pressure but shows a progressive decline with age. When compared with age corrected normal values, stroke distance was increased by $51 \%$ in 13 anaemic patients $(p<0.001)$ and by $43 \%$ in 12 pregnant women $(p<0.001)$. It was decreased by $14 \%$ in 15 hypertensives $(p<0.05)$, by $31 \%$ in 12 patients with atrial fibrillation ( $p<0.01)$, and by $43 \%$ in 13 patients with cardiac failure $(p<0.001)$. Stroke distance was normal in 16 patients convalescing from uncomplicated myocardial infarction.

Measurement of aortic blood velocity and its derivatives by itself, without determination of aortic size, meets the need for a simple non-invasive method of assessing cardiac output.

Early coronary thrombolysis with intravenous BRL 26921: a double blind placebo controlled study

$$
\begin{aligned}
& \text { M Been, D P de Bono, F E Bolton, A L Muir, W S } \\
& \text { Hillis, R Hornung } \\
& \text { Royal Infirmary,-Edinburgh, and Stobhill General } \\
& \text { Hospital Glasgow }
\end{aligned}
$$

The efficacy of intravenous BRL 26921, a "protected" streptokinase-plasminogen complex, in producing acute coronary reperfusion was assessed in a double blind placebo controlled trial. Thirty two patients within three hours of the onset of a first transmural myocardial infarction entered the study. Fourteen had anterior and 18 inferior infarcts. Half of each group received active and half placebo treat- ment. Reperfusion was assessed by coronary arteriography 90 minutes after injection of $30 \mathrm{mg}$ BRL 26921 or placebo (albumin). Coronary vessels were classified as reperfused or occluded without knowledge of the agent administered or the results of coagulation tests. Every patient who received BRL 26921 had patent vessels in the "infarct region" on angiography. All nine inferior infarct patients who received placebo had an occluded right coronary (7) or circumflex coronary (2) vessel. Five of seven patients in the anterior group had total occlusion of the left anterior descending coronary artery. Radionuclide ejection fraction at 10 days was significantly better in the anterior infarct group receiving active treatment $(37(7) \%)$ compared with placebo (23(5)\%), $\mathrm{p}<0.05$. There were two serious but non-fatal bleeding complications in the "active" group and two "late" deaths, both in the placebo group.

\section{Coronary reperfusion following single dose intravenous BRL 26921}

W S Hillis, R S Hornung, F G Dunn

Department of Materia Medica and Cardiology, Stobhill General Hospital, Glasgow

BRL 26921 is an inactive complex of acylated streptokinase and plasminogen with marked fibrin binding capacity. The active compound is produced by deacylation. Previous studies have shown successful coronary artery reperfusion following intracoronary administration within eight hours following acute myocardial infarction. This open study examined its efficacy using the intravenous route. BRL 26921 (30 $\mathrm{mg}$ ) was given intravenously to 29 patients ( 23 male, six female, age range 44-71 years) within six hours of acute myocardial infarction (mean $2 \mathrm{~h} 58 \mathrm{~min}$ ). Selective coronary arteriography was performed between 60 and 90 minutes following intravenous administration to confirm reperfusion. Anterior descending artery reperfusion occurred in 11 of 12 anterior myocardial infarctions (92\%). Vessel patency was confirmed in four of four circumflex and 16 of 17 right coronary vessels in the inferior infarct group (94\%). Reperfusion resulted in reversal of the acute electrocardiographic changes of infarct with a reduction in the sum of the ST segment elevation from 16(10) (mean (SD)) to $5(4) \mathrm{mm}(\mathrm{p}<0.001)$. There was an early peak of creatine phosphokinase release when compared with matched controls. Reperfusion arrhythmias occurred between 30 minutes and 2 hours after intravenous therapy and included idioventricular rhythm, ventricular extrasystoles, and 
reversal of heart block. There were no associated significant adverse effects.

These results confirm the efficacy of BRL 26921 to obtain reperfusion early in acute myocardial infaction.

Single dose intravenous thrombolysis in acute myocardial infarction: a non-invasive evaluation of a streptokinase-plasminogen activator complex (BRL 26921)

B D Vallance, J C Rodger, P W Macfarlane Departments of Medicine, Hairmyres Hospital and Monklands District General Hospital, Lanarkshire, and University Department of Medical Cardiology, Glasgow Royal Infirmary, Glasgow

We report a non-invasive evaluation of the coronary thrombolytic effect of an acylated streptokinaseplasminogen mixture (BRL 26921) administered in a single intravenous dose. Thirty four patients with acute myocardial infarction were investigated; all entered the study within three hours of the onset of symptoms. Sixteen were treated with $20 \mathrm{mg}$ intravenous BRL 26921 followed by a heparin infusion; the remainder formed the control group. Mean creatine phosphokinase activity before and at 12 and 24 hours after treatment was 129(73), 1941(1500), and 1397(814) IU/l respectively. The corresponding values in the control group were 156(19), 1703(852), and 1821(926) IU/1. Two ECG leads were recorded continuously. Computer analysis showed that at two hours ST segment amplitude was $28 \%$ of its initial value in the treated patients compared with $79 \%$ in the controls. Significant ventricular arrhythmias occurred in 15 treated and 13 control patients; the mean times to the first of these arrhythmias were 142 and 422 minutes in the treated and control groups respectively. Arrhythmias, early creatine phosphokinase peaking, and early reduction of ST segment elevation are accepted markers of reperfusion. All of these were present in 11 of the treated group but in only one of the controls. We conclude that BRL 26921 administered intravenously as in this study can produce coronary thrombolysis.

Angiographic assessment of treatment with intravenous single high dose streptokinase in early severe cardiac infarction

D B O'Keeffe, D P Nicholls, Patricia Morton, J G

Murtagh, M E Scott

Cardiac Unit, Belfast City Hospital, Belfast
Fifty consecutive patients (46 male, mean age 56) with early (mean $2 \mathrm{~h} 50$ min pain) severe cardiac infarction (mean Peel Index 14.6) received 1.5 million units streptokinase by intravenous injection over 30 minutes. Coronary artery reperfusion was identified on ECG criteria 20 to 135 minutes (mean $62 \mathrm{~min}$ ) later with an open infarct-related artery shown by angiography, in $84 \%$ of cases. The artery stayed occluded in the remaining $16 \%$. Mean time from onset of pain to coronary reopening was 198 mins. In these severe early infarcts, hospital mortality was $8 \%$ (three coronary reocclusions, one mesenteric embolus). There were no haemorrhagic complications needing transfusion within 48 hours of treatment. In those with coronary reperfusion the left ventricular ejection fraction (EF mean $51.4 \%$ ) was significantly better than in those whose artery stayed closed (EF mean $39.6 \%$ ) $(p<0.05)$, while the numbers with raised left ventricular end diastolic pressure in each group (35\% vs $67 \%$ ) were not significantly different $(p=0 \cdot 12)$. Thrombolysis was more likely to fail when patients were treated later $(170 \mathrm{~min}$ vs $212 \mathrm{~min}, \mathrm{p}<0.05)$ after the onset of infarct pain. Reperfusion arrhythmias were frequent but generally trivial, although ventricular fibrillation occurred in one case.

In conclusion, single high dose intravenous streptokinase treatment was followed by coronary reperfusion in $84 \%$ of cases and proved free of serious haemorrhagic complications. The earlier the treatment was applied the better the chance of coronary reopening. In hospital mortality may have been improved, and left ventricular function was significantly better in those patients whose affected coronary artery was reopened.

\section{Down's syndrome and complete atrioventricular septal defect: natural history}

\section{E A Shinebourne, C Bull, M L Rigby \\ Brompton Hospital, London}

Despite advances in surgical technique, repair of the complete atrioventricular septal defect carries a substantial early mortality and the risk of significant ongoing disability due to left atrioventricular valve dysfunction. Any evaluation of the place of surgery as a long term investment for a child with a complete atrioventricular septal defect must take into account the natural (unoperated) history of the condition. At the Brompton Hospital, London, since 1972 we have encountered 62 patients with Down's syndrome and complete atrioventricular septal defect without right ventricular outflow tract obstruction, on whom no 
surgery has been performed. Operations have been carried out on six patients during this period. Despite medical treatment with digoxin and diuretics, seven died in infancy during the phase of left to right shunting. Nevertheless, after this period mortality and usually morbidity decreased with the advent of pulmonary vascular disease. Actuarial survival at 15 years was $76 \%$ and for those who survived to 1 year of age, actuarial survival at 15 years was $87 \%$. These figures compare favourably with published surgical series and it seems likely that short and medium term survival of children with complete atrioventricular septal defect and Down's syndrome will be better without surgery. In the longer term, the life expectancy of patients with Down's syndrome, even without cardiac disease, is curtailed when compared with the normal population.

\section{Cardic malformation in spontaneous abortions}

\section{M Gerlis}

Cardiac Research Unit, Killingbeçk Hospital, Leeds

Although chromosomal abnormalities and external malformations are known to be very common in spontaneous abortions there does not appear to be any information on the frequency of cardiac malformations in such material. The subject was investigated by detailed examination of the hearts of 309 spontaneously aborted fetuses under the age of 24 weeks' gestation, by gross dissection, microdissection, or histological serial sections. Very early specimens, in which cardiogenesis was incomplete, were not included. Forty five instances of cardiac malformations were found (about 15\%). There was a bimodal relationship between the incidence of cardiac malformation and both maternal age and parity, and there was a higher incidence in summer conceptions but no apparent association with maternal blood groups. There were more male fetuses and they had proportionately more malformed hearts than the females. There was a marked inverse relationship with fetal age; in early fetuses, under $25 \mathrm{~mm}$ (about 9 weeks gestation), cardiac malformations were present in $68 \%$. A wide variety of morphological anomalies was encountered, ranging from simple to multiple and complex; some examples are illustrated. No cardiac malformations occurred in 72 threatened abortions which went to full term delivery.
Patient status five years or more after anatomic correction of transposition of the great arteries

R Radley-Smith, M H Yacoub

Harefield Hospital, Harefield, Middlesex

Between October 1975 and August 1984, 93 patients have undergone anatomic correction of transposition of the great arteries (TGA) at Harefield Hospital. Seventy six patients (82\%) survived operation, and 14 have been followed up for more than five years (mean 71 months). Nine patients aged 2 months to 10 years (mean 29 months) had TGA with additional large ventricular septal defect (group A) and underwent a single stage correction. Five patients aged between 4.5 and 21 (mean 9.6) months with simple TGA (group B) had a two stage operation. With a follow up of 65-99 (mean 71) months, there have been two late deaths in group A, one 18 months after operation for progressive pulmonary vascular disease and the other at 3 years of a cerebrovascular accident. The 12 surviving patients are asymptomatic and not on any medication. Holter monitoring has been performed both early and late in all patients. One patient in group B has remained in complete heart block since operation, but all other patients are in sinus rhythm. Routine recatheterisation and angiography have been performed at least once in all patients. One patient had a supravalve pulmonary gradient of $90 \mathrm{~mm} \mathrm{Hg}, 4.5$ years after correction and required reoperation. Left ventricular function, coronary artery growth, aortic root size, and anastomotic sites have all been evaluated both angiographically and by sequential echocardiogram. No significant abnormalities were found. Left ventricular ejection fraction was between 55$83 \%$ (mean $67 \%$ ) on angiography. It is concluded that anatomic correction gives satisfactory clinical and haemodynamic results five years or more after operation.

Balloon valvotomy and angioplasty in congenital heart disease: successes and failures

\section{G A H Miller}

Cardiac Department, Brompton Hospital, London

Balloon valvotomy or balloon angioplasty was successful in avoiding or delaying surgery in 13 of 16 patients with congenital heart disease. In 10 patients aged between 10 days and 7 years balloon pulmonary valvotomy was successful in all but two. In the eight successful cases right ventricular pressures averaged $70.5 \mathrm{~mm} \mathrm{Hg}$ before and $38 \mathrm{~mm} \mathrm{Hg}$ after the proce- 
dure. Gradients were reduced from an average of $51.5 \mathrm{~mm} \mathrm{Hg}$ to $15.6 \mathrm{~mm} \mathrm{Hg}$. The gradient was not reduced in a neonate with critical pulmonary stenosis and dysplastic valve. In a second patient failure was due to use of too small a balloon $(10 \mathrm{~mm})$; the gradient was reduced to $4 \mathrm{~mm} \mathrm{Hg}$ at a subsequent attempt when an $18 \mathrm{~mm}$ balloon was available. Recatheterisation in (so far) five patients at between 13 and 22 weeks demonstrates no significant change $(<5 \mathrm{~mm}$ $\mathrm{Hg}$ ) from immediate post valvotomy values in four and a further $(15 \mathrm{~mm})$ fall in right ventricular pressure in one patient suggesting that the benefit is retained, at least in the short term. In a patient aged $2 \frac{1}{2}$. yrs with complete transposition and previous Mustard's operation balloon angioplasty successful relieved severe obstruction at the lower limb of the baffle; to our knowledge, it is the first use of this technique to overcome this complication. Balloon angioplasty was clinically successful in a patient aged $11 / 2$ years with coarctation and abolished the gradient in another aged $3 \frac{1}{2}$ years with recoarctation. Finally detachable silicone filled balloons successfully obliterated several pulmonary arteriovenous fistulae causing severe arterial desaturation in a child aged 4 years. Balloon angioplasty failed to achieve dilatation of multiple peripheral pulmonary artery stenoses in a 2 year old. In a 13 year old with double outlet right ventricle and previous Rastelli operation failure to manipulate the catheter into the pulmonary artery frustrated attempts to dilate a severely stenosed homograft valve.

\section{Comparison of ECG gated computed tomography and left ventricular angiography in the measurement of left ventricular function}

C J Foster, T Sekiya, I Isherwood, W C Brownlee Department of Cardiology, Manchester Royal Infirmary, and the Department of Diagnostic Radiology, University of Manchester, Manchester

Twenty one studies were performed in 19 patients with ischaemic heart disease to compare ECG gated computed tomography with left ventricular angiography in the measurement of left ventricular volumes and ejection fraction. Gated computed tomographic scans each $1 \mathrm{~cm}$ in thickness were obtained at $1 \mathrm{~cm}$ intervals to image the entire left ventricle during intravenous infusion of contrast medium. End systolic and end diastolic areas were measured at each scan level to obtain the end systolic and end diastolic volumes, and from these ejection fraction was calculated. Angiography was performed in the $40^{\circ}$. right anterior oblique projection, and end systolic and end diastolic volumes were calculated using Kennedy's method. From these the ejection fraction was calculated. Comparison of end diastolic volumes revealed no significant difference between the two methods $(p=0.794)$. The coefficient of correlation was 0.74 . Computed tomography significantly overestimated the end systolic volume $(p<0.001)$ but the coefficient of correlation was $\mathbf{0 . 8 9}$. Computed tomography significantly underestimated the ejection fraction $(p<0.001)$ but the coefficient of correlation was $\mathbf{0 . 7 6}$. The results of this study indicate that ECG gated computed tomography is a useful non-invasive method for measuring left ventricular volumes and ejection fraction.

\section{Predicting the outcome of left ventricular aneurysmectomy by phase analysis of equilibrium radionuclide angiography (ERNA)}

O Ormerod, R Barber, D Stone, E Wraight, $M$ Petch Department of Nuclear Medicine, Addenbrooke's Hospital, and the Cardiac Unit, Papworth Hospital, Papworth Everard, Cambridge

Some patients show improvement in left ventricular function following resection of scar tissue (aneurysmectomy) but others do not. The former can be difficult to identify from contrast angiography. Computer processing of ERNA data allows measurement of both the amplitude and timing (phase) of wall movement and is well suited to the identification and quantification of paradoxical wall movement (dyskinesis). We have used this technique to study nine patients selected for aneurysmectomy by conventional angiographic criteria.

Patients were divided into those with dyskinesis (phase $>90^{\circ}$ and amplitude $>20 \%$ of maximum) (group $\mathrm{A}, \mathrm{n}=4$ ) and those with akinesis (amplitude $<20 \%$ ) (group $B, n=5$ ). Before operation group A had a lower global resting ejection fraction than group

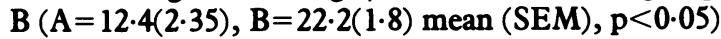
and a higher spread of phase on contractile myocar$\operatorname{dium}\left(A=31.8^{\circ}(4 \cdot 9), B=22.6^{\circ}(1.8), \mathrm{NS}\right)$. After operation there was an increase in the ejection fraction in group A $(29.9(2.1) \quad p<0.05)$ but not in group B $(21.9(3.0))$ and a greater fall in phase spread in group A $(17 \cdot 4(1 \cdot 35))$ compared with group B (20.2(2.6)). The change in ejection fraction correlated with the change in the amount of dyskinesis $(r=0.82, p<0.01$, $\mathbf{n}=9$ ).

The outcome of aneurysmectomy can be predicted 
from the extent of dyskinesis in the aneurysm but not by the ejection fraction or the function of the contractile segment. This suggests that dyskinesis, demonstrated by combined phase and amplitude analysis of ERNA, may be closer to the definition of a functional aneurysm than that of contrast angiography.

\section{Development of a miniature radiation detector for monitoring left ventricular volumes}

\author{
A Lahiri, J C W Crawley, R I Jones, M J Bowles, E B \\ Raftery \\ Northwick Park Hospital and Clinical Research \\ Centre, Harrow, Middlesex
}

The NaI scintillation probe (nuclear stethoscope) has been traditionally use for monitoring beat to beat left ventricular volumes from technetium-99m labelled blood pool. This instrument cannot be used for continuous monitoring of ambulatory subjects because of its size. Solid state mercuric iodide $\left(\mathrm{HgI}_{2}\right)$ has a theoretical advantage in that it has a higher stopping power for gamma rays, for the same volume, that $\mathrm{NaI}$ and does not require a photomultiplier tube. We have developed a miniature $\mathrm{HgI}_{2}$ detector $(0.5 \mathrm{~mm} \times 65$ $\mathrm{mm}^{2}$ ) which we have connected via an external amplifier to the computer of a nuclear stethoscope (Bios Inc). In 41 patients, undergoing nuclear probe assessment of left ventricular function, we were able to measure the left ventricular ejection fraction with both detectors. The ejection fraction measurements ranged from $31 \%$ to $82 \%$, and over this wide range of values we obtained an equation of: $y=9.44+0.84 x$ : $r=0.94$ for $n=41(p<0.001)$. The sensitivity of $\mathrm{HgI}_{2}$ detector compared with the $51 \mathrm{~mm}$ thick $\mathrm{NaI}$ detector was $55 \%$. The solid state $\mathrm{HgI}_{2}$ detector has now been adapted for continuous monitoring of left ventricular time activity curves at the bedside. This development opens up a new field of haemodynamic monitoring of left ventricular function with the possibility of extension to full ambulatory subjects.

ECG gated digital subtraction fine-catheter aortography in evaluation of coronary bypass grafts

R Hayward, G Hunter, R Emanuel

Departments of Imaging and Cardiology, Middlesex Hospital, London

Seven patients with recurrent chest pain have been studied three days to seven months after vein bypass grafting by conventional coronary and graft angiography, and by ECG gated digital subtraction angiography (DSA). DSA involved ascending aortic contrast injection $(30 \mathrm{ml}$ iohexol at $15 \mathrm{ml} / \mathrm{s})$ via a $4 \mathrm{FG}$ pigtail catheter. Images were acquired in arrested respiration at 0,300 , and $600 \mathrm{~ms}$ from the $R$ wave. Grafts were assessed as present or occluded; run off as absent (0), poor (1), moderate (2), or good (3), by two independent observers examining DSA images and angiograms. There were 18 grafts in seven patients, two being shown occluded. This status was correctly identified from DSA by both observers. Of the 16 patent grafts, run-off grading was such that the total score assigned by both observers using conventional angiography was $30 / 48$. Using DSA, run off was scored $29 / 48$ and $27 / 48$ by respective observers. In 6/7 patients visualisation of the native coronary anatomy was also achieved.

In terms of graft patency determination, ECG gated arterial DSA offers $100 \%$ sensitivity and specificity and the potential for deployment on an outpatient basis.

\section{Mechanisms of atrial flutter and conversion using atrioversion}

\section{A D Cunningham, J A Kennedy}

Department of Clinical Physics and Bio-Engineering, Glasgow, and Cardiac Department, Western

Infirmary, Glasgow

This study evaluated a new technique of electrical stimulation, atrioversion, in the treatment of atrial flutter (AFL). Atrioversion uses a single constant current impulse, with pulse width (mean $250 \mathrm{~ms}$ ) longer than the flutter cycle length. Rapid atrial pacing is widely used to convert AFL; a review of 22 papers shows that conversion to sinus rhythm occurs in $48 \%$ of cases. In 65 cases of AFL, atrioversion produced sinus rhythm in $53 \%$. Selection of a suitable atrial stimulation site affects the success rate. In a type I site, conventional $2 \mathrm{~ms}$ pulse width stimuli delivered during flutter produce return cycles which are not fully compensatory; longer return cycles define type II sites. For type I and II sites, conversion rate to sinus was $72 \%$ and $48 \%$; the difference was significant $(p<0.01)$. Conversion for type I sites was higher than for rapid atrial pacing. In five patients, the common form of flutter was converted to the uncommon form, with different $P$ wave axis, by delivering extrastimuli; in six cases, type I responses were found at different atrial sites, separated in activation time by long 
enough to rule out an ectopic focus. These results lead us to conclude that AFL is due to a macrore-entry circus movement. With careful selection of atrial site, atrioversion has a high success rate in the conversion of atrial flutter.

\section{Abnormalities of the peripheral circulation and respiratory function in patients with severe heart failure}

\section{A J Cowley \\ University Hospital, Nottingham}

To investigate the peripheral circulatory and respiratory abnormalities which occur in patients with heart failure we have measured forearm and calf blood flow and calf venous tone, before and after upright exercise, and respiratory gas exchange during exercise in a group of 26 patients with severe heart failure. Compared with a group of normal subjects the patients had reduced limb blood flow at rest and the response of limb blood flow to upright exercise was also abnormal. The patients developed a smaller increase in calf blood flow after exercise $(p<0.001)$ and also a smaller reduction in blood flow in the non-exercising forearm $(p<0.001)$. There was a significant correlation between the maximum exercise duration of the patients and calf blood flow both after exercise $(r=0.642$, $\mathrm{p}<0.01)$ and at rest $(\mathrm{r}=0.526, \mathrm{p}<0.02)$. The patients were also venoconstricted at rest and after exercise $(p<0.01)$. Compared with another group of control subjects the patients had a higher minute ventilation during exercise $(p<0.03)$ and a reduced oxygen consumption $(p<0.005)$. This latter suggests the early development of anaerobic metabolism during exercise.

A measurement of limb blood flow and respiratory gas exchange are useful non-invasive ways of assessing patients with severe heart failure.

Ambulatory ST segment monitoring: the mechanisms and pathophysiology of nocturnal myocardial ischaemia

A A Quyyumi

National Heart Hospital, London

The role of ambulatory ST segment monitoring and the incidence, mechanisms, pathophysiology, and therapy of nocturnal angina were investigated. The sensitivity (70\%) and specificity (97\%) of ambulatory
ST monitoring in detecting coronary disease $(n=100)$ was similar to exercise testing but the patients' history of pain had a poor correlation with the frequency of ambulatory ST changes. Nocturnal resting ST changes occurred in $15 \%$ of patients; the majority had severe coronary artery disease. Analysis of momentary changes demonstrated that heart rate increases preceded ST depression in the majority of nocturnal episodes in patients with coronary disease $(n=5)$. Polygraphic sleep studies $(n=10)$ showed that these increases in heart rate were a result of arousal, bodily movement, rapid eye movement (REM) sleep, or apnoea during sleep. Detailed pathological examination of coronary arteries showed no anatomical differences between patients with and without nocturnal ST segment changes and spasm causing total luminal occlusion could not occur in the arteries. Reduction of basal heart rate with atenolol $(n=15)$ reduced the frequency $(p<0.01)$ of nocturnal angina when compared with pindolol, which did not reduce resting heart rate owing to its partial agonist activity. Revascularisation with coronary artery bypass grafting $(n=46)$ reduced the frequency of both daytime and nocturnal ST changes.

Thus an increase in myocardial oxygen demand is important in the precipitation of nocturnal resting myocardial ischaemia in patients with coronary artery disease and its reduction produces significant relief in daytime and nocturnal ischaemia.

\section{Improvement in exercise induced ST segment depression early after myocardial infarction}

R I Jones, A Lahiri, E B Raftery

Northwick Park Hospital and Clinical Research Centre, Harrow, Middlesex

Forty five male patients with a mean age of 53 years (range 36-67) underwent maximal treadmill exercise testing a mean of 8 days after their first uncomplicated transmural myocardial infarction (MI) when taking no cardiovascular medications. A 12 lead ECG was recorded during each minute of exercise, and directly measured brachial arterial blood pressure was recorded. ST segment depression of $>1 \mathrm{~mm} 0.08 \mathrm{~s}$ after the $\mathrm{J}$ point constituted a positive response. Twenty three patients developed a positive ECG response during their initial exercise test. The mean (SD) maximal heart rate for these patients was $127(18)$ beats/min and increased to $139(14)$ beats/min at week $8(\mathrm{p}<0.001)$. Exercise time increased from 6.7(2.1) mins to $9.4(3.9)$ mins $(p<0.001)$ and maximal rate pressure product (RPP) from $172(33)$ to $218(46)$ 
$(\mathrm{p}<0.001)$. Mean ST segment depression at maximal exercise decreased from $1.9(0.8) \mathrm{mm}$ at week 1 to $1 \cdot 1(0.6) \mathrm{mm}$ at week $8(\mathrm{p}<0.01)$ with a corresponding fall in exercise induced ST segment elevation in reciprocal leads from $1 \cdot 6(1 \cdot 1) \mathrm{mm}$ to $1 \cdot 0(1 \cdot 2) \mathrm{mm}$. Six patients with a positive response during the first study did not show a positive response during the second study despite achieving a higher workload. For the 16 patients with a positive ECG response on both occasions heart rate at the onset of $1 \mathrm{~mm}$ ST segment depression increased from $110(12)$ beats $/ \mathrm{min}$ to 121(14) beats/min $(\mathrm{p}<0.01)$, RPP from $150(24)$ to $184(28)(p<0.01)$, and exercise time from 3.9(1.3) mins to $6.4(2.4)$ mins $(p<0.001)$ between the two studies.

These data suggest that exercise induced ST segment depression may decrease during the early weeks following myocardial infarction, which may account for some of the observed differences in the prognostic significance of ST segment depression at this time. The timing of exercise testing after myocardial infarction may be of fundamental importance.

Exercise testing following myocardial infarction in patients under 55 years: a test of limited value?

I Peart, L Seth, Catherine Albers, R J C Hall Royal Victoria Infirmary, Newcastle upon Tyne

ST segment depression or angina or both during post myocardial infarction exercise testing is reported reliably to predict the extent of coronary artery disease. As such information is important in the management of young patients following myocardial infarction we have studied 100 consecutive patients under 55 years (range 32-54 years, mean 46.6 years) surviving an acute myocardial infarction. They had treadmill exercise tests using a modified Bruce protocol at three weeks (submaximal) and six weeks (maximal) after infarction. During exercise testing 16 lead precordial maps were recorded in addition to standard 12 lead ECG's. All patients had coronary angiography. Forty patients had single vessel disease and 60 patients multivessel disease. Thirty three (55\%) patients with multivessel disease had a positive exercise test (angina, ST depression $\geqslant 1 \mathrm{~mm}$ or blood pressure fall $\geqslant 20 \mathrm{~mm}$ $\mathrm{Hg}$ ) six weeks following infarction compared with 22 $(37 \%)$ at three weeks $(p<0.05)$ using the standard 12 lead ECG. The 16 lead precordial map did not improve detection of multivessel disease. The sensitivity, specificity, and predictive value of a positive six week postinfarction exercise test for multivessel disease was $55 \%, 70 \%$, and $73 \%$ respectively. Twenty five $(81 \%)$ of the 31 patients who had angina during the exercise test six weeks postinfarction had angina on follow up compared with $14(20 \%)$ of the 69 who did not $(p<0.001)$.

Thus exercise testing at six weeks following myocardial infarction in patients under 55 years is of limited value in detecting multivessel disease but is a reliable predictor of angina on follow up.

\section{Reproducibility of ischaemic abnormalities resulting from maximal exercise tests performed predischarge and six weeks after infarction}

C E Handler, E Sowton

Guy's Hospital, London

Symptom limited treadmill exercise tests were performed predischarge and six weeks after myocardial infarction (MI), without complications, in 64 patients (mean age 55 years) to assess the reproducibility of abnormalities of prognostic value. There was a significant lack of reproducibility among those patients with angina $(p<0.005)$ and an abnormal blood pressure (BP) response $(\mathrm{p}<0.05)$. ST segment depression and elevation and ventricular arrhythmias were relatively reproducible. There was a significant increase in the frequency of an ischaemic response from the early to the later test $(p<0.01)$ associated with a significantly longer exercise duration $(p<0.001)$ and a greater mean maximal heart rate $(p<0.001)$ and heart rate-systolic BP double product $(p<0.001)$. Eight patients were not retested at six weeks. Six of these had complications after leaving hospital; two reinfarcted, one of whom died, and four had unstable angina. All six patients had had an abnormal predischarge test $(p<0.05)$.

We conclude that the majority of patients who develop immediate complications after infarction can be identified with a predischarge exercise test. Because exercise induced ST segment depression and elevation are reproducible, patients who develop these abnormalities at the predischarge test do not, for prognostic reasons, need retesting at six weeks. Angina and an abnormal BP response, however, are highly variable, and in these patients a repeat test may be useful. 
Coronary morphology and ventricular function in patients with positive exercise tests following myocardial infarction

\section{P Murray, L B Tan, M Salih, Sally Derry, R G Murray, W A Littler \\ Department of Cardiovascular Medicine, University of Birmingham and East Birmingham Hospital, Birmingham}

Coronary morphology and left ventricular (LV) function were investigated in 76 survivors of acute myocardial infarction with positive predischarge exercise tests. Patients were selected by ST depression in a 12 lead treadmill test (73 patients) or by an abnormal exercise blood pressure (BP) response ( 3 patients). Coronary anatomy and LV performance were determined at catheterisation. Multiple vessel disease was demonstrated in 60 of the $73(82 \%)$ patients with ST abnormality remote from the site of infarction. A single locus of ST abnormality based on ECG lead groupings was present in 46 patients. Dual loci were present in 27 patients, of whom 25 had multiple vessel disease (7 double vessel disease, 15 triple vessel disease). Seventy eight of the 101 (77\%) loci of ST abnormality corresponded to coronary arteries with significant stenosis, and 65 (83\%) of those diseased vessels served LV segments with normal wall motion. Twenty seven loci of ST abnormality corresponded to normal vessels. In 18 of these 27, exercise induced ST elevation in alternate loci or arteriographic collateral circulation or both suggested a possible coronary steal mechanism. An abnormal BP response was elicited in 17 patients, all of whom had multivessel disease compared with 24 patients with a BP increase $>20 \mathrm{~mm}$ $\mathrm{Hg}$, of whom nine had single vessel disease. Mean LV ejection fraction was significantly lower in the former group $(0.45(0.04))$ compared with the latter $(0.58(0.04) ; \mathrm{p}<0.01)$.

A positive predischarge exercise test conferred a high risk of multiple vessel disease. The locus of ST abnormality was related to disease in individual vessels which supplied preserved LV segments. Abnormal BP response indicated multiple vessel disease and impared global LV performance.

Painless myocardial ischaemia: sequence and magnitude of left ventricular volume changes

G J Davies, S Chierchia, W Bencivelli, F Crea, T

Pratt, J Crow, M Morgan, A Maseri

Department of Cardiology, Royal Postgraduate

Medical School, London

Left ventricular volume (precordial counting of technetium-99m labelled blood pool) and the electrocardiogram were continuously recorded in 12 patients with angina at rest during 11 spontaneous (two painful) and 12 ergometrine induced (nine painful) attacks of acute myocardial ischaemia, characterised by ST segment elevation in 13 (four painful) and ST segment depression in 10 (seven painful). All ischaemic episodes were accompanied by progressive left ventricular dilatation, reduction of stroke volume and ejection fraction. These changes preceded the onset of diagnostic ST segment change and, when present, the onset of pain. The total duration of ventricular dilatation was greater in painful episodes than in painless episodes. Rate of change of volume was less in ergometrine induced episodes than in spontaneous episodes and less in those with ST segment depression than in those with ST segment elevation. Nevertheless, there was no significant difference in rate of volume change between painful and painless episodes. The maximum change in end systolic volume, end diastolic volume, stroke volume, and ejection fraction was $133(50) \%$ (mean (SD)), 38(8.0)\%, $-28(17) \%$, and $-32(8.7) \%$ respectively for painful episodes. It was $110(27) \%, 26(12) \%,-25(12) \%$, and $-25(6) \%$ respectively for painless episodes.

Asymptomatic ST segment changes are accompanied by the typical left ventricular volume changes of acute myocardial ischaemia but differences in magnitude or initial rate of ventricular dilatation do not appear to be sufficient to explain the occurrence of pain.

Implications of the routine use of beta blockade after myocardial infarction in England and Wales

\section{R A Greenbaum \\ Royal Free Hospital, London}

Long term beta blockade after myocardial infarction has been suggested significantly to improve one year survival. The purpose of this study is to estimate the effect and cost if this policy were applied nationally. During 1981, in England and Wales, 100360 patients were admitted to hospital following acute myocardial infarction. Of these, 75240 were discharged. Two trials have shown a mortality for treatment groups of 6.8\% (timolol) and 3.6\% (propranolol) in the first year after infarction. The corresponding placebo groups had mortalities of $10.7 \%$ and $6.0 \%$. This has been interpreted as a $\mathbf{3 0 \%}$ reduction in mortality. Alternatively, this could be seen as an improvement from an average $92 \%$ survival without treatment to $95 \%$ with beta blockade. Nationally, there is a potential saving 
of 2300 lives. This contrasts with a national mortality, in 1981, of 107000 from myocardial infarction. For each patient the cost of one year's treatment with timolol (10 mg twice daily) is $£ 77.53$ and with propranolol (80 mg thrice daily) $£ 69$. Treatment of all 75240 survivors would cost $£ 5833060$ with timolol and $£ 5190430$ with propranolol. The cost per life saved would be $£ 2540$ for timolol and $£ 2260$ for propranolol. In practice fewer lives could be saved because both studies indicated that $18 \%$ of infarct survivors had contraindications to treatment with beta blockade.

It is concluded that in England and Wales approximately 1900 lives might be saved in the first year after infarction at a cost of about $£ 4553000$.

Can good risk patients with acute myocardial infarction be discharged after four days?

A Evans, G McKenzie, K Salathia, D McC Boyle Cardiac Unit, Ulster Hospital, Dundonald, and Department of Community Medicine, Queens University, Belfast

Reducing the length of hospitalisation of patients with acute myocardial infarction would release resources for other patient care. We report a randomised study to determine if very short stay (four days) versus long stay (seven days) management of patients with low risk affects the outcome at three months looking at $(a)$ mortality and $(b)$ cardiac events (death and non-fatal reinfarction) as end points. Non-invasive prognostic index identified $45.5 \%$ of patients to be low risk. Data were examined by "intention to treat" and by explicitative analysis. Using "intention to treat" analysis, 187 patients were allocated to four day discharge and 177 to seven day discharge. Mortality at three months was $8.0 \%$ and $4.0 \%$ and cardiac events $9.1 \%$ and $5.6 \%$ respectively. By explicitative analysis, 137 patients were discharged on the fourth day, and 155 on the seventh day. Mortality at three months was $5.8 \%$ and $1.3 \%$ respectively. Cardiac events occurred significantly more often in the early discharge patients $(10.5 \%)$ than in the late discharge group (3.2\%).

Heart rate variability during acute myocardial infarction: relation to ventricular fibrillation

D McAreavey, J M M Neilson, D C Russell Departments of Cardiology and Medical Physics, Royal Infirmary, Edinburgh

Complex autonomic effects occur during acute myocardial infarction, may relate to pathogenesis of ventricular fibrillation (VF), and may be reflected by patterns of RR interval variability. Detailed analysis has been performed of electrograms from 14 cases of acute myocardial infarction developing VF and 15 cases of acute infarction (six anterior, nine inferior) without VF. Electrograms were processed from tape by computer in five minute segments to determine $R R$ intervals, sequential $R R$ interval differences (excluding ectopic beats), and calculate 10th to 90th centile sample ranges. Distinctive patterns of $R R$ variability observed include $(a)$ sinus arrhythmia, $(b)$ oscillation at about $0.1 \mathrm{~Hz},(c)$ bursts of sinus tachycardia (1-2 minutes), with or without ventricular arrhythmias, and $(d)$ absence of any visible oscillation. Abolition of patterns $(a)$ and $(b)$ was observed after administration of atropine. During the five minute period preceding $\mathrm{VF}$, the prevalence of pattern (d) was significantly greater (nine cases/14) than was found in comparable 5 min periods (10/74) in 15 cases not developing VF $(p<0.001)$ and mean $R R$ interval shorter $(680(38) \mathrm{ms}$ vs 750 (22) ms. RR difference range was greater in inferior (49(6) $\mathrm{ms})$ than anterior $(20(2) \mathrm{ms})$ infarction $(p<0.001)$. Although mean $R R$ interval was more prolonged during inferior $(803(24) \mathrm{ms}, \mathrm{p}<0.05)$ than anterior infarction $(726(22) \mathrm{ms})$ no correlation was found between $R R$ difference range and heart rate $(r=0.09)$.

Results suggest that the heart may be subject to parasympathetic influences which can be independent of heart rate during acute inferior infarction and that withdrawal of vagal tone with enhanced sympathetic activity may immediately precede VF.

\section{A comparison of bepridil with amiodarone in the conversion of established atrial fibrillation}

M S Perelman, E Rowland, D M Krikler Cardiovascular Unit, Royal Postgraduate Medical School, Hammersmith Hospital, London

While conventional drug treatment of established atrial fibrillation rarely reestablishes sinus rhythm amiodarone converts approximately $40 \%$ of patients. Bepridil, introduced for angina, has both fast and slow channel blocking properties and possesses antiarrhythmic properties. We have compared bepridil with amiodarone in 18 patients with poorly controlled and established atrial fibrillation (mean ventricular rate $>100 / \mathrm{min}$; duration $>1$ month). The ventricular rate was assessed at rest and during a standard treadmill exercise test (Naughton protocol) performed weekly over three weeks on bepridil $(200,400$, and 
$600 \mathrm{mg} /$ day) and at two and four months on amiodarone (400 mg/day). During bepridil 9/14 patients returned to sinus rhythm, while during amiodarone $4 / 13$ converted. In the 10 patients who received both agents sequentially bepridil converted six and amiodarone four. In those patients remaining in atrial fibrillation amiodarone reduced the ventricular response more at rest (baseline: 145(38), amiodarone: 101(6), bepridil: 117(32) (mean (SD)); on exercise control was similar. The ventricular rate in atrial fibrillation was not further slowed by increasing bepridil above $200 \mathrm{mg} /$ day, but more patients returned to sinus rhythm $(200 \mathrm{mg}, \mathrm{n}=2: 400 \mathrm{mg}$, $\mathrm{n}=6: 600 \mathrm{mg}, \mathrm{n}=9$ ). In $8 / 9$ patients, returning to sinus rhythm on bepridil, the QTc was $>440$ ms. This prolongation of the QTc was dose related, and in two patients on $600 \mathrm{mg} /$ day the return to sinus rhythm was associated with a QTc of $>600 \mathrm{~ms}$ and torsade de pointes which degenerated into ventricular fibrillation in one.

While bepridil proved useful for the conversion of established atrial fibrillation, its effect on the QTc and the associated ventricular arrhythmias are likely to restrict its use and dosage.

\section{Clinical use of intravenous flecainide for acute onset of tachyarrhythmias}

A W Nathan, K J Hellestrand, D E Ward, R S

Bexton, A J Camm

St Bartholomew's and St Georges' Hospitals, London

Intravenous flecainide $(2 \mathrm{mg} / \mathrm{kg}$ ) was given during acute onset tachycardias to 75 patients. Twelve had atrial flutter and 12 atrial fibrillation (including three with pre-excitation). Thirty two had junctional arrhythmias (atrioventricular (AV) re-entrant tachycardia in 20, AV nodal tachycardia in 12) and 19 had ventricular tachycardia. In the following, ventricular rates (1 standard deviation from the mean in beats per minute) before and after flecainide are shown in brackets. Atrial flutter was terminated in $2 / 12$ patients (112(14) to 87(7); $p<0.001)$. Atrial fibrillation was stopped in 11/12 cases (159(11) to 121(9); $p<0.05)$. Atrioventricular tachycardia was reverted in $17 / 20$ patients $(188(34)$ to $142(27) ; p<0.001)$. IntraAV nodal tachycardia was stopped in 10/12 cases (175(36) to $134(20) ; p<0.001)$. Ventricular tachycardia was reverted in $15 / 19$ patients (176(13) to $115(14)$; $\mathrm{p}<0.01$ ). In those with junctional tachycardias, termination was in the retrograde limb (accessory pathway or "fast" AV pathway) in 25 and anterograde limb (AV node) in only two. Four had proarrhythmic effects. One, with AV nodal tachycardia, developed ventricular fibrillation, and another with ventricular tachycardia degenerated into fibrillation; both had received other antiarrhythmic drugs, and two patients with atrial flutter developed fast ventricular rates. All four responded to electrical therapies. Minor side effects included oral parasthesiae, transient dizziness, and visual blurring.

Flecainide is an effective and relatively safe drug for the termination of recent onset paroxysmal atrial, junctional, and ventricular tachyarrhythmias of all types except atrial flutter.

\section{D-sotalo- the clinical electrophysiological effects of a new class III antiarrhythmic agent \\ E Rowland, E J Perrins, R M Donaldson, A F Rickards \\ National Heart Hospital, London}

The clinical importance of drugs with class III antiarrhythmic activity has been established. Sotalol, in addition to its beta blocking action, has class III activity, thought to be a property of the dextroisomer. We have studied the electrophysiological effects of the pure dextroisomer (d-sotalol) in five patients undergoing investigation for arrhythmia; three had paroxysmal ventricular tachycardia, one had AV nodal re-entry tachycardia, and one paroxysmal atrial flutter. D-sotalol $0.75 \mathrm{mg} / \mathrm{kg}$ was administered intravenously over 10 minutes during sinus rhythm after baseline electrophysiological measurements had been obtained; these included $90 \%$ repolarisation time derived from a monophasic action potential recording. The electrophysiological variables were reevaluated 10-20 minutes after the end of infusion. There was a small decrease in mean heart rate from 88 to 80 beats/min, prolongation in surface QTc interval (428(15) to 442 (16) ms (mean (SD)), but no change in intracardiac conduction intervals. AV nodal refractoriness was unchanged. Prolongations in both atrial (208(9) to $227(15) \mathrm{ms}$ ) and ventricular (228(12) to 250 (24) $\mathrm{ms}$ ) effective refractory periods occurred together with a lengthening of the $90 \%$ repolarisation time (260(12) to $284(24) \mathrm{ms})$. In two patients with VT inducible prior to d-sotalol, VT could not be induced after drug administration: in the other patient nonsustained VT (cycle length $220 \mathrm{~ms}$ ) was induced prior to the infusion, slower but sustained VT (cycle length $260 \mathrm{~ms}$ ) being induced afterwards.

This study is the first to demonstrate the class III antiarrhythmic action of d-sotalol; the absence of an 
AV nodal action suggests that the immediate effects are not achieved by beta adrenoceptor antagonism. The electrophysiological profile suggests that d-sotalol will be an important alternative to amiodarone for the management of arrhythmias.

\section{Xamoterol, an oral agent for the treatment of sinoatrial disease?}

\section{F L Tseu, C A Morley, A F Mackintosh Killingbeck Hospital, Leeds}

Xamoterol (Corwin) is a highly selective beta adrenoceptor antagonist with $45 \%$ intrinsic sympathomimetic activity; this combination suggests a role in the treatment of sinoatrial disease. Ultilising a randomised double blind crossover study over four weeks with placebo run-in and washout, xamoterol $200 \mathrm{mg}$ twice daily was compared with placebo. At the end of each week, patients were assessed by a semiquantitative symptom checklist and Holter monitoring outside hospital. The tapes were analysed blind for the hourly $R R$ interval distribution, the number of pauses $>1.74 \mathrm{~s}$, the longest pause, and the presence of atrial arrhythmias. Twelve patients (eight men, four women; aged 50 to 79 years, mean 64.8 years) were studied. Eleven were symptomatic; nine were referred for consideration of permanent pacing. Eight had atrial tachycardias in addition to sinus bradycardia or atrial pauses. Xamoterol reduced the range of RR intervals over 24 hours. The drug was of clinical benefit in 10 patients. Six showed a reduction in the number and maximum duration of pauses. Three reported symptomatic improvement associated with an increase in mean heart rate. The other patient also had an increase in mean heart rate but was unable to describe her symptoms. Six patients are now on longterm therapy. One of the 12 patients showed no improvement; another with paroxysmal atrial flutter deteriorated.

The drug treatment of sinoatrial disease can be assessed outside hospital in a wide range of patients. In many patients xamoterol could provide simple but effective therapy.

Effect of successful percutaneous transluminal coronary angioplasty on myocardial perfusion and function

A Singh, S Chandler, M F Shiu

Departments of Cardiology and Nuclear Medicine, Queen Elizabeth Medical Centre, Edgbaston, Birmingham
Thallium 201 scintigraphy ( $T 1201)$ and technetium angiography (Tc-99m) were performed on 19 patients before and six weeks after successful treatment by percutaneous transluminal coronary angioplasty (PTCA). The degree of reversible myocardial ischaemia at exercise and rest was assessed on Tl 201 scintigrams using a four point ischaemia score $(0$, normal, 3, severe abnormality) with images obtained in four standard views and the myocardium divided into five anatomical segments. Successful dilatation was achieved in 23 vessels in the 19 patients. Symptomatic improvement was maintained in 17 patients at six weeks and effort tolerance improved from 97(6) to $111(6) \mathrm{J}(\mathrm{p}<0.02)$. Reversible ischaemic score (RIS) in the whole group improved from 3.0(0.9) to $0.6(0.2)$ $(p<0.05)$. Left ventricular ejection fraction (LVEF) at rest remained unchanged. LVEF at exercise increased from $0.40(0.03)$ to $0.52(0.02)(p<0.001)$. Seven patients (group 1) had a significant ( $p>0.05$ ) increase in exercise LVEF. The RIS in this group prior to PTCA was $7.8(1.8)$ whereas in 12 patients without improvement in exercise LVEF the RIS was $0.8(0.03)(p<0.001)$. There were no differences in either groups in numbers of vessels treated or site of lesion.

This study showed that improvement in left ventricular systolic function following successful PTCA was more likely in patients with substantial degrees of reversible ischaemia before the procedure.

PTCA in unstable angina: comparison with stable angina

P J Quigley, G F Gearty, B J Maurer, M J Walsh Royal City of Dublin Hospital, Dublin, Eire

Unstable angina is a recognisable clinical entity with a significant risk of subsequent myocardial infarction and death. Percutaneous transluminal coronary angioplasty (PTCA) was performed in 25 patients presenting with unstable angina and the early and late results analysed in order to assess its efficacy in the treatment of this condition. Results were compared with a similar group of 25 patients with stable angina in whom PTCA was also performed. The incidence of single, double, and triple vessel disease was identical in each group. Technical success was achieved in 23 out of $27(85 \%)$ attempts in the unstable group and in 14 out of $27(52 \%)$ attempts in the stable group $(p<0.05)$. Vessel occlusion occurred in nine patients overall, necessitating emergency bypass surgery in four. Evidence of myocardial infarction was found in 
three (12\%) patients in each group, and one patient in the unstable group subsequently died. Twenty patients in the unstable group have been followed up for a mean period of $\mathbf{7 . 6}$ months. Nineteen patients are symptom free while one (5\%) has recurrent angina.

These results compare favourably with published reports on unstable angina treated medically or surgically and demonstrate the effectiveness of PTCA in this condition.

\section{Clinical effects of prolonged balloon inflation during coronary angioplasty and their moderation by intracoronary nitroglycerin}

\section{Amende, G Herrmann, W P Hood Jr, R Simon, P R Lichtlen \\ Hanover Medical School, Hanover, West Germany}

Prolonged balloon inflation time $(30-60 \mathrm{~s})$ has been shown to improve results of coronary angioplasty, but the extent of the ischaemia so produced has not been well defined. We studied the haemodynamic and electrocardiographic effects of prolonged inflation during angioplasty of the proximal left anterior descending coronary artery in 29 patients, before and after administration of $0.2 \mathrm{mg}$ nitroglycerin (NTG) through the distal balloon catheter port. There were no significant changes in heart rate or aortic pressure during balloon inflation, with or without NTG. Before NTG, baseline pulmonary wedge pressure was $11 \cdot 1(6.2) \mathrm{mm} \mathrm{Hg}$ and after NTG was 7.4(4.3) $\mathrm{mm} \mathrm{Hg}$ $(p<0.001)$. Wedge pressure at $60 \mathrm{~s}$ inflation was 18.3(7.4) $\mathrm{mm} \mathrm{Hg}$ before NTC and $14.5(6.8) \mathrm{mm} \mathrm{Hg}$ after $(p<0.001)$. The time to an increase in wedge pressure $\geqslant 5 \mathrm{~mm} \mathrm{Hg}$ was $31.6(13.9) \mathrm{s}$ before and $40.8(16.3) \mathrm{s}$ after the drug $(p<0.005)$. The time to appearance of ST segment elevation or depression $>0.1 \mathrm{mV}$ in lead I or aVL was $25.2(12.1) \mathrm{s}$ before NTG and $34.5(16.2)$ s afterward $(p<0.001)$. Six patients with an increase in wedge pressure $\geqslant 5 \mathrm{~mm}$ $\mathrm{Hg}$ during balloon inflation had no detectable electrocardiographic alterations.

In conclusion, pulmonary wedge pressure rises in response to balloon inflation during coronary angioplasty and is a more sensitive indicator of ischaemia than aortic pressure or electrocardiographic changes in standard leads. Intracoronary nitroglycerin delays the appearance of the rise in wedge pressure and limits its extent; it also delays the onset of ischaemic ST segment changes. Monitoring of wedge pressure and administration of intracoronary NTG improve the safety of employing an extended inflation period during coronary angioplasty.
Successful percutaneous coronary angioplasty in totally occluded coronary arteries

A Singh, M F Shiu

Department of Cardiology, Queen Elizabeth

Hospital, Birmingham

In a consecutive series of 70 procedures, percutaneous transluminal coronary angioplasty (PTCA) was performed in eight patients with total occlusion. Occlusion occurred up to eight months between diagnostic angiography and PTCA and was associated with increased anginal symptoms but no $Q$ wave infarction. The procedure was successful with no complications in seven patients, with angiographic evidence of vessel patency up to nine months later. One patient had successful recanalisation of the obstructed vessel but required emergency bypass grafting following PTCA of another vessel. All patients had symptomatic improvement and four were pain free at follow up between three and 14 months. Thallium 201 scintigraphy before and after PTCA showed regression of fixed defects in four patients and normalisation of defects in two.

In conclusion, coronary artery occlusion unassociated with a $Q$ wave infarct can be treated by PTCA with substantial clinical benefit and no increased risks.

\section{Should angioplasty be attempted for occluded} vessels?

A F Rickards, K M Fox, R Canepa-Anson, J Perrins National Heart Hospital, London

In the course of 107 consecutive angioplasty procedures in 103 patients, an attempt was made to recanalise mechanically an occluded vessel on 15 occasions. Two patients had suffered an acute myocardial infarct with vessels that were presumed to have occluded within the previous two hours. In these individuals recanalisation was successful and antegrade flow reestablished with prompt relief of pain and reversal of ECG changes. Thrombus was not observed in the affected vessel and streptokinase was not used. Follow up angiography revealed continued patency of the vessels without significant obstruction. In 13 chronically occluded vessels, guide wire passage was successful in five with balloon passage in three and successful dilatation in one. At restudy the only successfully dilated vessel had asymptomatically reoccluded. In the group as a whole the primary success rate for dilatation of stenoses was $79 \%$ with evidence of 
myocardial damage occurring in seven, of which five developed $Q$ wave infarction. One patient was treated by immediate surgery. There were no deaths. No complications occurred in relation to attempted recanalisation of occluded vessels.

In sharp contrast to dilatation of stenoses, recanalisation of chronically occluded vessels with currently available techniques is unsuccessful, uncomplicated, and unrewarding.

\section{Outcome of early surgery following coronary angioplasty}

\section{S Norell, $R$ Balcon \\ London Chest Hospital, London}

Nineteen (27\%) of 69 patients undergoing angioplasty underwent immediate coronary surgery, six planned for continuing angina after failed angioplasty. The remaining 13 were operated upon because of complications: they were dissection in four, occlusion in five, continuing severe spasm in two, tamponade in one, and in one other detachment of a guide wire tip. The angioplasty was for a lesion in the anterior descending in eight $(62 \%)$ patients, the right in four $(31 \%)$, and the circumflex in one (8\%). This was a similar distribution to the 56 patients without complications. There was one early postoperative death due to extensive anterior myocardial infarction, and there was ECG evidence of new infarction in three others. Nuclear left ventricular angiograms were performed in 16 of the 18 patients surviving immediate surgery after a mean of 27 months. These were compared with the cineangiograms performed prior to attempted angioplasty. Only two patients showed evidence of a new left ventricular wall motion abnormality, the remaining 14 having normal left ventricular contraction both at rest and after a maximal bicycle exercise.

Prompt surgery after angioplasty complications is usually successful with good symptomatic relief and without left ventricular damage. The incidence of important surgical complications in these low risk patients is, however, high. These are not always included in reported angioplasty series being "lost" in the "emergency surgery group."

\section{Are changes in left ventricular function during exercise related to the extent of coronary artery disease? A study using first pass radionuclide angiography with gold-195m}

J L Caplin, D S Dymond, J O'Keeffe, W Flatman, S O Banim, R A J Spurrell
Cardiology Department, St Bartholomew's Hospital, London

To examine if sequential changes in left ventricular ejection fraction during exercise could quantify the extent of coronary disease, 25 patients underwent first pass radionuclide angiography with gold-195m ( $\mathrm{t}^{1 / 2}$ $30.5 \mathrm{~s}$ ). Seven patients had normal coronaries (group A), 11 had single or double vessel disease (group B), and seven had triple vessel disease (group C). Ejection fraction was measured at rest, three minutes, six minutes, peak, immediately after, and 2 minutes after exercise. Ejection fractions did not fall in group A during exercise. In group B it fell from $58(10) \%$ (mean (SD)) at rest to $51(10) \%$ at three minutes, and $47(10) \%$ at peak (both $\mathrm{p}<0.05$ vs rest); after exercise it rose to $64(8) \%$ ( $<<0.05$ vs rest). In group $\mathrm{C}$, ejection fraction fell from $57(12) \%$ at rest to $42(9) \%$ at three minutes, and $35(8) \%$ at peak (both $\mathrm{p}<0.05$ vs rest). It did not rise above rest after exercise. Seventy per cent of group C and $45 \%$ of group B had abnormal exercise responses at three minutes, and all group $C$ and $75 \%$ of group $B$ were abnormal at peak. The rate of change of ejection fraction from rest to peak was $+0.6(0.9) \% / \mathrm{min}$ for group $\mathrm{A},-2 \cdot 1(1.7) \% / \mathrm{min}$ for group $B(p<0.01)$, and $-4.7(2.7) \% /$ min for group C $(\mathrm{p}<0.001$ vs group $\mathrm{A}, \mathrm{p}<0.05$ vs group $\mathrm{B})$, but there was overlap between groups A and B and groups B and $\mathrm{C}$.

In conclusion, $(a)$ falls in left ventricular ejection fraction at submaximal exercise help identify patients with severe coronary disease, $(b)$ the rate and duration of ejection fraction depression are related to extent of coronary disease but the degree of overlap precludes definite anatomical classification in individuals, and $(c)$ in patients with single or double vessel disease, rapid or sustained falls in ejection fraction may suggest similar amounts of muscle at risk as in triple vessel disease.

Three dimensional quantification of left ventricular wall motion by ECG gated blood pool emission tomography

S R Underwood, S Walton, P J Laming, P H Jarritt, P J Ell, R W Emanuel, R H Swanton

Middlesex Hospital and Medical School, London

In equilibrium radionuclide ventriculography, imaging of the left ventricle is restricted principally to a single projection to avoid overlap with the right ventricle. This problem can be overcome using the new 
technique of ECG gated blood pool emission tomography, in which information from multiple projections is reconstructed into tomographc sections through the intracardiac blood pools. This study establishes a simple method for displaying the three dimensional data so obtained, determines a normal range for ejection fraction in all regions of the left ventricle, and compares wall motion in abnormal subjects with that determined by $x$ ray contrast ventriculography. Regional ejection fraction was plotted on a colour coded circular image, in which the apex was represented in the centre, the base around the circumference, and all other parts of the ventricle were represented in between. The image was divided into 15 segments, and normal segmental ejection fraction was defined as within two standard deviations of the mean in a group of 10 normal subjects. In 25 subjects with coronary artery disease, motion of the anterior, apical, and inferior walls agreed in every case with the right anterior oblique contrast ventriculogram, but in 12 of these, the three dimensional ejection fraction image showed abnormal septal motion in addition. In all 12 there was disease of the left anterior descending coronary artery.

ECG gated blood pool emission tomography thus provides an accurate quantitative assessment of left ventricular wall motion in three dimensions and has significant advantages over conventional planar techniques.

\section{Measurement of valvular regurgitation by first pass radionuclide angiocardiography}

\section{S Walton, S R Underwood, P J Ell, R H Swanton, R W Emanuel \\ Departments of Cardiology and Nuclear Medicine, Middlesex Hospital and Medical School, London}

A novel technique is described for the measurement of valvular regurgitation. After bolus administration intravenously of $740 \mathrm{MBq}$ of $99 \mathrm{~m}$ technetium pertechnetate activity is sampled over the lung and left ventricle and flow calculated from the resulting indicator dilution curves. In mitral or aortic regurgitation tracer passing to and fro across the valve is sampled more than once in the ventricle but not the lung. The area under the ventricular, but not the pulmonary, curve will be overestimated, and thus flow will be underestimated. This effect should be proportional to the degree of regurgitation. In 24 normals the ratio of pulmonary to left ventricular flow ( $P f / L V f)$ had a gaussian distribution with a mean value of 1.0 and standard deviation (SD) of $0 \cdot 1$. The mean Pf:LVf ratio of 14 further subjects without valvular regurgitation but with reduced left ventricular ejection fraction $(0.45-0.17$, mean 0.35$)$ was also unity. All values differed $2 S D$ from the mean of the normals. All of 20 subjects with valvular regurgitation did, however, manifest abnormally low Pf:LVf values. The distribution in these subjects was skewed to the left, reflecting varying degrees of valvular regurgitation with a modal value of 0.7 and a range of $0.4-0.8$.

The ratio Pf:LVf can be used to quantify valvular regurgitation. It is simple to perform, non-invasive, and independent of impaired left ventricular contraction.

\section{Magnetic resonance cine imaging of the human heart}

\section{J P R Jenkins, J W Waterton, H G Love, Zhu Xiao Ping, I Isherwood, D Rowlands \\ Departments of Diagnostic Radiology and Cardiology, University of Manchester, Manchester}

A new method which allows five magnetic resonance sections to be produced at the same anatomical level at predetermined points in the cardiac cycle has been developed. This has been achieved by modifying an ECG gate spin echo sequence. A further five sections can be interleaved by repeating the sequence and employing a gating delay. The five to 10 sections produced are then displayed in a cine mode. A Picker International superconducting magnet operating at 0.26 Tesla has been used to collect the anatomical data. The cine sequence has been obtained on an independent IGE system. Five volunteers and four patients have been studied. By using the coronal plane for cine display direct comparison can be made with left ventricular angiography. Myocardial thinning, localised areas of dyskinesia, and valvular motion are clearly visualised. Subjective estimates can be made of left ventricular ejection fraction and further work is in progress to determine objective measures of cardiac function. A signal observed in the great vessels and pulmonary circulation should permit further study of blood flow.

Nuclear magnetic resonance imaging in hypertrophic cardiomyopathy and hypertension

\author{
M Been, D M Kean, M A Smith, R H B Douglas, \\ A L Muir \\ NMR Unit, Royal Infirmary, Edinburgh
}

Proton nuclear magnetic resonance (NMR) permits 
visualisation of cardiac structures, and with measurement of the spin-lattice relaxation time $\left(T_{1}\right)$ tissue characterisation may be possible. We have explored the capability of calculated $T_{1}$ images, obtained from inversion recovery and saturation pulse sequences using a low field $(0.08 \mathrm{~T})$ resistive magnet to differentiate between normal and hypertrophied hearts. Eleven patients with hypertrophic cardiomyopathy diagnosed either by echocardiography or by cardiac catheterisation were compared with 11 age matched volunteers and 11 patients with hypertension and electrocardiographic evidence of left ventricular hypertrophy (LVH). The ratio of septal to LV thickness in patients with LVH measured by NMR was $0.8(0.2)(S D)$ and by echocardiography $0.9(0.3)$. In patients with hypertrophic cardiomyopathy the ratio was $1.5(0.8)$ and $1.6(0.6)$ respectively. Septal area in volunteers was $2.7 \mathrm{~cm}^{2}$ compared with $4.6 \mathrm{~cm}^{2}$ and $6.2 \mathrm{~cm}^{2}$ for patients with LVH and hypertrophic cardiomyopathy. Corresponding areas for LV free wall were $3.7,6.3$, and $6 \cdot 1 \mathrm{~cm}^{2}$. For all groups septal $T_{1}$ was higher than free wall $T_{1}$.

NMR allows two dimensional estimation of septal and free wall mass without the limitations of echocardiography. Gated NMR will improve accuracy and precision and allow more subtle differences in $T_{1}$ to be observed.

\section{Magnetic resonance imaging of the human heart}

H G Love, J P R Jenkins, I Isherwood, D Rowlands Departments of Diagnostic Radiology and Cardiology, University of Manchester, Manchester

ECG gated and non-gated magnetic resonance studies have been performed in 22 volunteers and 30 patients with proven cardiac disease. The contours of the ventricular and atrial chambers together with major vessel morphology can be identified on non-gated images. Image quality improved significantly during ECG gated studies and allowed identification of many additional features. The transverse axial plane permitted evaluation of ventricular and atrial size and of myocardial thickness. The morphological features of each chamber and their associated valves were clearly identifiable. The mid-coronal plane approximates best to the right anterior oblique left ventricular angiogram. Using this plane, particularly in the cine mode, it has been possible to identify localised areas of wall thinning and to assess regional wall motion. It is concluded that non-gated magnetic resonance imaging is a useful method of examining the great vessels and may be used to identify all cardiac chambers. ECGgated magnetic resonance, however, significantly improves image quality enabling the internal morphology of the heart to be examined in considerably greater detail. Areas of myocardial thinning are readily detectable and estimates of left ventricular wall motion can be made. Magnetic resonance imaging was performed on a Picker International superconducting magnet operating at 0.26 Tesla.

\section{Ventricular arrhythmia surgery based on a new mapping}

\author{
R W F Campbell, P Kertes, C Griffiths, D S Reid, \\ C J Hilton \\ Department of Cardiology, Freeman Hospital, \\ Newcastle upon Tyne
}

In 17 patients with recurrent medically intractable life threatening ventricular tachycardia or ventricular fibrillation, arrhythmia surgery was based on electrogram mapping in sinus rhythm. The patients were aged 32 to 65 years; 16 had had a myocardial infarction (three weeks to 15 years previously) and one had a "normal" heart. In the ischaemic patients the mean ejection fraction was $25 \%$ (range 13-44\%). Epicardial and endocardial maps (53 and 36-48 points respectively) were obtained in sinus rhythm in all patients. Mapping during the arrhythmias was possible in only seven patients. Fragmentation (electrogram duration $>100 \mathrm{~ms}$ ) in sinus rhythm was used to identify areas for endocardial resection. Fragmentation always extended beyond any aneurysm resection line but was not always associated with visually abnormal endocardium. In the one patient with a "normal" heart, fragmentation was present throughout the left ventricle and almost all the left ventricular endocardium was resected. Resection was supplemented by coronary artery bypass grafting in 10 patients, and aneurysmectomy in 12 patients. The operative mortality was $30 \%$ (four pump failure, one infection). Durng a mean follow up of 13.8 months (range 2 months to 3.2 years) there have been no arrhythmia recurrences or late deaths.

Thus effective surgery for refractory ventricular tachycardia and ventricular fibrillation can be based on electrogram characteristics determined during sinus rhythm. Arrhythmia surgery is now possible in situations where peroperative arrhythmia maps are unobtainable (for example, ventricular fibrillation). Morever, as this technique is applied in haemodynamically stable conditions, the risks of preoperative myocardial damage may be minimised. 
Exercise electrophysiology study of arrhythmias

J C P Crick, P Holt, C Bucknall, P V L Curry, E Sowton

Guy's Hospital, London

Eighteen patients with tachycardias have been investigated with programmed stimulation studies during erect maximum exercise. Fifteen patients had leads placed in the right atrium, coronary sinus, or right ventricle and two leads were used in three patients. In 12 cases an active fixation screw-in lead was used in the right atrium. All leads were inserted percutaneously via a subclavian vein. In 12 of the $18(67 \%)$ patients the exercise study revealed arrhythmias which could not be induced at rest even following atropine. These arrhythmias included atrial fibrillation (seven), re-entry tachycardia (six), atrial flutter with block (one), and ventricular fibrillation. PR interval changes were also disclosed. In one patient with Wolff-Parkinson-White syndrome atrial fibrillation induced at rest was well tolerated but during exercise caused syncope. There were no complications from the studies. In 10 of the 18 cases the management was changed as a result of the exercise findings. In many patients it is no longer adequate for electrophysiological studies to be performed only at rest.

\section{Atrioversion-conversion of atrial flutter in man using a long constant current pulse}

\section{A D Cunningham, J A Kennedy \\ Cardiac Department, Western Infirmary, Glasgow}

This study evaluated a new technique of electrical stimulation, atrioversion, in the treatment of atrial flutter (AFL). Atrioversion uses a single constant current impulse, with pulse width (mean $250 \mathrm{~ms}$ ) longer than the AFL cycle length. Rapid atrial pacing is widely used to convert AFL; a review of 22 papers shows that conversion to sinus rhythm (SR) occurred in $48 \%$ of cases, and that a long duration of flutter or digitalisation or both lowered the success rate of rapid pacing. Atrioversion was used to treat 65 cases of spontaneous AFL in 59 patients ( 46 male, 13 female, mean age 54 years). Ten were converted using one to three conventional coupled extrastimuli (nine to SR, one to atrial fibrillation (AF)). In the remaining 55, atrioversion produced sinus rhythm in $28(53 \%)$. AF occurred in $20(36 \%)$, and in six (11\%) AFL persisted. Nine patients spontaneously returned to sinus rhythm from AF. SR was regained immediately in 37 of the 65 $(57 \%)$ cases, and overall in $47(72 \%)$. The most impor- tant factor affecting success rate was selection of a suitable atrial stimulation site. When conventional ms pulse width stimuli delivered during AFL produce return cycles which are not fully compensatory, a type I response is seen; longer return cycles define type II sites. For type I and II sites, immediate conversion to SR was $72 \%$ and $48 \%$; the difference was significant $(p<0.01)$. It is presumed that a type I site is closer to the origin of the flutter. Duration of flutter and digoxin were not shown to significantly affect conversion rate. Atrioversion has a high success rate in converting atrial flutter to sinus rhythm and, with optimal catheter positioning, is more successful than rapid atrial pacing $(p<0.015)$. Its usefulness in other arrhythmias is being evaluated.

\section{Repetitive ventricular responses in at risk patients following myocardial infarction: relationship to left ventricular function}

\section{W Pumphrey, J D Skehan, M T Rothman \\ The London Hospital, London}

Although ventricular function is accepted as the principal factor in determining survival following myocardial infarction, it remains to be established whether additional prognostic information may be obtained by studying rhythm abnormalities provoked by ventricular electrical stimulation. Such responses were studied within one month of infarction in 90 consecutive patients considered "at risk" because of a positive early submaximal exercise test. A positive electrical response was defined as $\mathbf{4}$ or more spontaneous ventricular beats after delivery of either single or extrastimuli at diminishing intervals to the right ventricle in sinus rhythm or at paced cycle lengths of 600 and $500 \mathrm{~ms}$. Eight of 48 patients with anterior and 11 of 42 patients with inferior infarcts had positive responses. These 19 patients with a positive response had a similar mean ejection fraction $(54.7(18.0) \%$ vs $46.9(17.6) \%)$ and regional ventricular wall motion score $(19.7(4.6)$ vs $19.8(6.0))$ compared with the 71 patients with a negative response (14 of whom had an ejection fraction of $<35 \%)$. Significant abnormalities on 24 hour ECG monitoring were found in five of 19 patients with a positive response compared with seven of 71 patients with a negative response. Four of 70 patients followed for a minimum of three months (mean 7-9 months) have died. All four patients had positive electrical responses, but only one had an abnormal 24 hour ECG. Nevertheless, these four patients had a lower mean ejection fraction, (26.6(12.4)\% vs $59.0(14 \cdot 1) \%)$ and higher mean wall 
motion score $(24.0(6.9)$ vs $20 \cdot 8(5 \cdot 1)$ compared with those patients with positive electrical responses who have survived. This suggests that positive electrical responses are not related to ventricular function and may indicate a poor prognosis in those patients with severe ventricular dysfunction.

Effects of iloprost, a stable prostacyclin analogue, on exercise tolerance and platelet aggregation in patients with stable angina pectoris

\section{R Bugiardini, C Gridelli, D Ferrini, M Galvani, P Puddu, S Lenzi Clinica Medica II, University of Bologna, Italy}

Prostacyclin and its analogues combine in one molecule two properties-coronary dilatation and anti-platelet action-which seem extremely promising for the treatment of stable angina pectoris. Accordingly we assessed the effects of iloprost, a chemically stable compound which has been shown to have prostacyclin mimetic activities, on exercise tolerance and platelet aggregation in 24 patients with effort angina and proven critical $(\geqslant 70 \%)$ coronary artery disease. Upright bicycle ergometer testing ( $25 \mathrm{~W}$ increments every $2 \mathrm{~min}$ ) was performed during iloprost $(6 \mathrm{ng} / \mathrm{kg}$ per min) or placebo infusion and 30 and 60 minutes later, using a crossover randomised single blind protocol. Samples for measurements of adenosine diphosphate (ADP) induced platelet aggregation were obtained before and throughout the study. Compared with placebo, intravenous iloprost administration consistently $(p<0.001)$ prolonged exercise duration (mean (SD): 511(142) vs 436(146) s) and time to onset of significant $(0.1 \mathrm{mV})$ ST segment depression (368(140) vs 285(122) s). Also, angina occurred at a higher $(\mathrm{p}<0.001)$ heart rate $(138(11)$ vs $130(13)$ beats $/ \mathrm{min})$ and rate pressure product $(26.2(3.0)$ vs $\left.23.5(2.9) \times 10^{3} U\right)$. Similar differences in exercise duration and time to onset of ST segment depression were observed when comparing placebo with iloprost 30 minutes after infusion. Changes in platelet aggregation paralleled those in exercise duration and a marked decrease in ADP induced platelet aggregation during exercise was found concurrently with iloprost infusion (by 78(12)\%) and 30 minutes later (by $52(16) \%)$. No significant differences in exercise tolerance and platelet aggregation were observed between placebo and iloprost 60 minutes after their infusions.

We conclude that $(a)$ iloprost administration improves exercise tolerance in patients with stable exertional angina pectoris; and $(b)$ improvements are independent of changes in the major determinants of myocardial oxygen demand and are associated with markedly reduced platelet aggregation. Benefits in exercise tolerance might be due to diminished release of vasoactive platelet derivatives and increased myocardial perfusion.

\section{Effects of placebo on indices of exercise induced myocardial ischaemia - an objective analysis}

R S Kohli, N S Khurmi, A Lahiri, M Bowles,
E B Raftery
Northwick Park Hospital and Clinical Research Centre, Harrow, Middlesex

It is known that placebo can influence the frequency of anginal attacks favourably, but there is no clear consensus on exercise tolerance. This has considerable implications for the ethics of placebo periods in clinical trials of antianginal drugs. We have analysed the effects of placebo in 150 patients (135 male, 15 female) aged 42-75 years with stable exertional angina pectoris who had participated in single and double blind studies. Multistage treadmill graded exercise testing (modified Chung's protocol), using online computer analysis of the electrocardiogram (Marquette CASE I), was performend after the patients had been on no treatment for at least two weeks and after two weeks of oral treatment with placebo three times a day. The leads monitored were CC5 and CM5 and recorded as 25:1 computer averaged complexes. The tests were performed at a controlled temperature between $20^{\circ} \mathrm{C}$ and $24^{\circ} \mathrm{C}$ on the same equipment between 09.00 and 11.00 . Although $20 \%$ of the patients showed subjective improvement, the objective measurements were not altered by placebo. The mean (SEM) exercise time untreated was $6 \cdot 0(0.2)$ minutes and with placebo was $6 \cdot 1(0 \cdot 2)$ minutes (NS). Similarly, the $1 \mathrm{~mm} \mathrm{ST}$ depression time was $4.0(0.2)$ minutes with no treatment and $4 \cdot 1(0 \cdot 2)$ minutes after placebo (NS) and the resting and maximal heart rates were $74(1)$ and $124(1)$ beats/min respectively untreated and 73(1) and $122(1)$ beats/min respectively after placebo treatment (NS). The placebo failed to show any effect on maximum ST segment depression. These results question the need for placebo control in antianginal drug trials which use exercise testing for the evaluation of effect. 
A modified Bruce protocol compared with the original

C J Reid, S Subhedar, R B Pridie

Harefield Hospital, Harefield, Middlesex

The Bruce protocol is widely used in exercise ECG testing for the recognition of myocardial ischaemia. It has the disadvantage of taking a considerable time to perform, and some patients stop prematurely before the age predicted heart rate has been achieved. Such tests are inconclusive. In an attempt to overcome these difficulties, the stages of the Bruce regimen were reduced from three minutes to one minute. A comparison was made of 25 patients undergoing the full Bruce protocol (B) and the same patients doing a modified exercise test (A) with similar increments of speed and gradient but progressing at one minute intervals. At least one hour elapsed between the two tests. Half the patients did A first and half B. Group $1(n=17)$ were on beta blocking drugs and group $2(n=8)$ were not. All patients who had a positive exercise test on $B$ also had a positive test on $\mathrm{A}$. The mean heart rate of group 1 rose from 57.2 to a maximum of 116.5 beats/min on $A$ and from 61.3 to 109.6 beats $/ \mathrm{min}$ on B. Group 2 maximum heart rate rose from 72.3 to 155.6 beats/min on A and from 69.3 to 138.6 beats/ min on $B$. The difference is not statistically different ( $p>0.01)$. The mean maximal systolic blood pressure response was not significantly different between the two protocols. The mean ST depression at maximum heart rate was $1.95 \mathrm{~mm}$ (range $0.5-2.6$ ) on regimen $A$ and $2.18(0.6-2.9)$ on $B$ in group 1 and $0.73(0.1-1.7)$ on protocol $A$ and $0.76(0.1-1.9)$ on $B$. The average duration of exercise on protocol $A$ was 4.9 mins and on B 8.6 mins. These results show that the modified protocol (A) is entirely comparable to the conventional Bruce protocol and much less time consuming.

\section{Elevated diastolic blood pressure response to exercise testing when coronary artery disease is suspected: an indication of severity}

F Akhras, J Upward, G Jackson

Cardiac Department, King's College Hospital, London

Our objective was to evaluate the significance of a rising diastolic blood pressure during exercise. One hundred and two consecutive patients with a history of chest pain underwent maximal symptom limited treadmill stress testing and coronary angiography. The diastolic blood pressure response to exercise was evaluated independently of ST segment change and systolic blood pressure (SBP). In the presence of a normal systolic blood pressure response an increase in DBP of $15 \mathrm{~mm} \mathrm{Hg}$ on at least two determinations during the same stage of exercise was considered abnormal. Of the 61 patients with normal DBP response, the ST segment change was positive for ischaemia in 25 . Seven of these had three vessel coronary artery disease. Of the 38 patients with an abnormal DBP response, 27 had a positive exercise ECG for ischaemia. Eleven patients had a negative exercise ECG. Of these, one had left main stem disease, seven had three vessel disease, and the remaining three had two vessel disease. In these 11 patients the diastolic blood pressure response to exercise was abnormal. ST segment analysis showed that patients with abnormal diastolic blood pressure had more ST segment depression than those with a normal diastolic blood pressure response $(1.48(0.17)$ vs $0.93(0.16), \mathrm{p}<0.05)$. In addition there were significantly more patients developing angina $(78 \%$ vs $31 \%)$ at a significantly reduced exercise time in the group of patients with an abnormal diastolic blood pressure when compared with those showing a normal response $(5 \cdot 3(1.8)$ vs $7.6(1.7)$ mins, $\mathrm{p}<0.001$ ).

Thus an abnormal diastolic blood pressure response to a maximal symptom limited treadmill stress test is a useful additional indicator of the severity of coronary artery disease and may be of special clinical value when the ST segment response is normal or borderline.

Long term follow up of ST segment reciprocal change in acute myocardial infarction: correlation with exercise testing and coronary angiography

F Akhras, J Upward, G Jackson

Cardiac Department, King's College Hospital, London

Our objective in this study was to evaluate the significance of ST segment "reciprocal" changes defined as $>1 \mathrm{~mm}$ in the ECG leads other than those reflecting acute myocardial infarction. Eighty five consecutive uncomplicated cases of acute myocardial infarction were studied prospectively with maximal symptom limited treadmill stress tests at two weeks and coronary angiography at six weeks post infarction. Forty six patients had inferior, 34 anterior, and five true posterior infarction. Of the 51 patients with reciprocal changes, $45(88 \%)$ developed exercise induced ST segment depression in areas remote from the infarction zone. At angiography all 45 were shown to have stenoses $>70 \%$ in at least two major vessels. 
Four patients had negative exercise ECGs and were subsequently shown to have single vessel coronary artery disease subtending their.infarct, and the remaining two patients had a false negative treadmill result. Of the 34 patients without reciprocal changes, $21(62 \%)$ had a negative treadmill stress test associated with single vessel disease, five had a positive stress test and multivessel disease, and one had a false negative stress test. The remaining seven patients had ST segment elevation without $Q$ wave formation in the reciprocal areas. Of these, six had positive stress tests and multivessel disease and one had a negative test and single vessel disease to the infarct area. At follow up (36 months) of the 51 patients with reciprocal ST segment change, 36 became symptomatic and 29 of these underwent coronary bypass surgery. By contrast, only seven of the 34 patients without reciprocal ST segment change developed symptoms and three of these underwent coronary revascularisation. Reciprocal ST segment depression at the time of acute myocardial infarction may identify patients with significant coronary artery disease and when positive appears to be as reliable as early post infarction treadmill stress testing in predicting the underlying coronary anatomy. Nevertheless, when negative, treadmill testing provides valuable diagnostic information.

The role of ambulatory ST segment monitoring in clinical practice
A A Quyyumi, Christine Wright, Lorna Mockus, K M Fox
National Heart Hospital, London

The role of $\mathbf{4 8}$ hour ambulatory monitoring was investigated in 100 consecutive patients with chest pain undergoing stress testing and arteriography. Patients were off antianginal medication. Nineteen per cent of patients with normal coronaries had reversible ST changes (specificity $77 \%$ ), of whom only $3.8 \%$ had proven coronary spasm. ST changes occurred in $70 \%$ of patients with coronary artery disease; $73 \%$ had positive stress tests. Three vessel disease was detected more efficiently than single vessel disease, but poor left ventricular function was more often associated with the absence of ST changes $(p<0.01)$. Ninety six rer cent had episodes of ST depression and $4 \%$ also had ST elevation. The frequency, duration, and magnitude of episodes were greater in patients with more severe disease and lower exercise tolerance in whom the heart rate at the onset of ST depression tended to be lower. Nocturnal ST changes occurred in $15 \%$ of patients (three vessel disease or left main stenosis), but a third did not complain of any rest pain. No correlation existed between the history of pain and either the severity of coronary artery disease or frequency of ST changes. Only $76 \%$ of patients with frequent pain ( $>1 /$ day) had ST changes, whereas $66 \%$ of patients with infrequent pain $(<1 /$ week) also had changes in 48 hours of monitoring. Painful ST changes (33\% of total) occurred with similar frequency in patients with varying severity of coronary artery disease. Thus the history was not a sensitive indicator of the frequency of underlying ischaemia (ST changes). If therapy is to be tailored to the type of angina (rest/exertional) ambulatory ST monitoring is desirable.

\section{Exercise testing and arrhythmia monitoring predicts triple vessel disease post infarction}

E D Bennett, D Mannering, D Ward, M Dancy Department of Medicine 1, St George's Hospital . Medical School, London

In order to investigate the associations between blood pressure (BP) response, time to onset of ST segment depression during exercise, and subsequent coronary anatomy routine exercise stress testing was undertaken in 93 patients three weeks post infarction. BP was measured at rest and post exercise. Thirty seven (40\%) patients had a positive test and underwent coronary angiography. The percentage rise in systolic BP on exercise was $27(2) \%$ for those with a negative test, and $27(4) \%$ and $23(6) \%$ for those with single and double vessel disease respectively. In marked contrast, those with triple vessel disease only increased BP by $5(2) \%(p \leqslant 0.005)$. Onset time to $1 \mathrm{~mm} \mathrm{ST}$ depression was $4.6(0.5) \mathrm{min}$ and $4.2(0.8) \mathrm{min}$ for single and double vessel disease compared with 2.1(0.4) min for those with triple vessel disease $(p \leqslant 0.01)$. In 65 patients, 24 hour ECG taping was undertaken eight days post infarction. The mean Lown score was $1.9(0.2)$ in those with a negative test, $3.0(0.4)$ for a positive test $(p \leqslant 0.01), 1.3(0.3)$ for single vessel disease, $2.6(0.7)$ for double vessel disease, and $3.2(0.3)$ for triple vessel disease $(p \leqslant 0.01)$.

Triple vessel disease post infarction relates to early onset of ST depression, poor exercise BP response, and significantly increased ventricular arrhythmias. 
Prognostic value of non-invasive investigations in the management of the post myocardial infarction patient

D P Murray, R G Murray, E Rafiqi, W A Littler Department of Cardiovascular Medicine, University of Birmingham, and East Birmingham Hospital, Birmingham

The ability of non-invasive techniques to predict further cardiac events and multiple vessel coronary disease was investigated in 46 apparent low risk survivors of acute myocardial infarction. Exercise electrocardiography (ETT), rest/exercise thallium-201 (Tl-201) scintigraphy, and rest/exercise radionuclide angiography (RNA) were performed prior to hospital discharge. At investigation, half were beta-blocked and thereafter all patients were given prophylactic beta blockade. Coronary arteriography was performed at three months post discharge. During a mean follow up of $12(4)$ months, 12 patients (26\%) experienced cardiac events (recurrent myocardial infarction, three patients; angina pectoris, 11 patients; coronary surgery, six patients). No patient died during the follow up period. Ten of the 12 were identified by T1201 scintigraphy (reversible image defect) or positive ETT, and seven displayed abnormal left ventricular exercise reserve. The predictive accuracy of Tl-201, ETT, and RNA for cardiac events was $87 \%, 76 \%$, and $57 \%$ respectively and was not influenced by beta blockade. T1-201 specificity for cardiac events (88\%) exceeded that of ETT (76\%) and RNA (56\%; $\mathrm{p}<0.01)$. The predictive accuracy of T1-201, ETT, and RNA for multivessel disease was $89 \%, 70 \%$, and $80 \%$ respectively. Nevertheless, arteriographic multivessel disease, although $100 \%$ sensitive, was a nonspecific predictor $(52 \%)$ for subsequent cardiac events.

Thus T1-201 scintigraphy appeared to be the technique of choice for the assessment of patients following myocardial infarction both in terms of subsequent cardiac events and predicting multiple vessel disease. The accuracy of the technique was not reduced by beta blockade.

Does intravenous loading with amiodarone hasten the appearance of an electrophysiological effect?

\author{
M S Perelman, E Rowland, W J McKenna, \\ D M Krikler \\ Cardiovascular Unit, Royal Postgraduate Medical \\ School, Hammersmith Hospital, London
}

The significant delay between starting oral amiodarone and the demonstration of an antiarrhythmic effect is an important problem when treating "malignant" arrhythmias. We have assessed whether intravenous loading will accelerate the appearance of an electrophysiological change. Eleven patients were randomly allocated to either intravenous or oral loading with amiodarone, given over 30 hours. A bipolar electrode was placed at the right ventricular apex and at least 24 hours later the baseline effective refractory period determined. Six patients received intravenous amiodarone by a constant infusion $(0.65$ $\mathrm{mg} / \mathrm{kg} / \mathrm{h}$ ): three into an antecubital vein and three into a central vein. Five patients received oral amiodarone $(0.85 \mathrm{mg} / \mathrm{kg} / \mathrm{h})$ given as divided doses $(10$ $\mathrm{mg} / \mathrm{kg} / 12$ hourly). The refractory period was determined at a constant cycle length $\left(S_{1}-S_{1}\right)$ of $500 \mathrm{~ms}$ at baseline and then serially at $6,12,18$, and 30 hours after starting amiodarone. At the same times amiodarone and desethyl amiodarone serum concentrations were measured. After oral amiodarone the refractory period did not increase until 30 hours $(p=0.022)$; with intravenous loading the refractory period was significantly increased at 18 hours $(p=0.032)$ and at 30 hours showed further lengthening $(p=0.0043)$. Two patients receiving intravenous amiodarone experienced nausea, while the three patients in whom the intravenous amiodarone was given into a peripheral vein developed thrombophlebitis necessitating a change of infusion site. One patient receiving oral amiodarone felt faint without a change in blood pressure.

While intravenous loading hastens the onset of an electrophysiological effect there is still a significant hiatus during which protection against arrhythmias is likely to be inadequate.

\section{What is "controlled atrial fibrillation"? \\ D W Pitcher, M Papouchado, M A James, J R Rees Bristol Royal Infirmary, Bristol}

Ambulatory 24 hour ECGs were recorded in 66 patients with chronic atrial fibrillation judged clinically to be adequately controlled. Recordings were analysed to assess fastest and slowest heart rates in each hour, duration of pauses, and the nature and frequency of ventricular arrhythmias. Maximum heart rates occurred during the day in all patients, ranging from 84 to 188 beats per full minute, and exceeding 150 beats/minute in 18 patients (27\%). Slowest heart rates ranged from 34 to 82 beats/ minute, occurred at night in 63 patients (95\%), and were less than 40 beats/minute in 12 patients (18\%). 
Pauses longer than $1.5 \mathrm{~s}$ occurred at night in 59 patients $(89 \%)$ and during the day in $35(53 \%)$. Pauses longer than $2.0 \mathrm{~s}$ were seen in 44 patients $(67 \%)$ and $13(20 \%)$ had pauses lasting between $3 \cdot 1$ and $4.0 \mathrm{~s}$. Only two patients had no ventricular arrhythmias. More than 500 ventricular extrasystoles per day were recorded in 38 patients (58\%). Twenty patients (30\%) had other ventricular arrhythmias including frequent couplets, bigeminy, and ventricular tachycardia. In 59 patients taking digoxin fastest heart rates, nocturnal bradycardia, length of pauses, and ventricular arrhythmias all correlated poorly with serum digoxin levels.

Thus in patients with clinically "controlled" atrial fibrillation ambulatory ECG recordings may show marked variations in heart rate despite apparently appropriate suppressant therapy. Nocturnal bradycardia and asymptomatic pauses of up to $4 \mathrm{~s}$ duration may occur without digoxin toxicity and do not warrant cardiac pacing. Ventricular arrhythmias are common without digoxin excess and are probably due primarily to the underlying heart disease.

\section{Ambulatory monitoring of atrial electrograms}

\author{
D W Davies, J C P Crick, P M Holt, P V L Curry, \\ E Sowton \\ Guy's Hospital, London
}

One of the limitations of conventional ambulatory ECG monitoring is the difficulty in reliably detecting the $P$ wave. Using a two channel ambulatory ECG recorder, we monitored the endocardial atrial electrogram obtained with a temporary bipolar screw-in lead in 18 patients and with a conventional temporary bipolar electrode positioned in the coronary sinus in two patients. A surface ECG lead (CM5) was recorded simultaneously on the second channel. Clear electrograms were obtained in all cases; initial mean amplitude 1.38 (range $0.7-2.8) \mathrm{mV}$ with final amplitudes of 1.1 (range $0.4-2 \cdot 2) \mathrm{mV}$. A mean initial injury current of 0.76 (range $0.3-2.1$ ) $\mathrm{mV}$ was seen in $17 / 18$ patients with the screw-in lead falling to 0.54 (range 0-2.4) $\mathrm{mV}$ after 24 hours. In nine patients with accessory AV conduction pathways, spontaneous arrhythmias were not seen during the recording period but the electrode was used to initiate and terminate reentry tachycardia under different autonomic settings. Tachyarrhythmias were seen in nine patients: four, atrial, four, ventricular, and one, Coumel. In all cases, useful or diagnostic information, not available from the surface ECG, was provided by the atrial electrogram. The electrode was used to terminate atrial flutter in two patients, Mobitz II AV block was seen in one patient in whom the electrode did not provide additional information whereas in another patient with apparent AV dissociation on the surface ECG the atrial electrogram revealed $P R$ alternans during sinus rhythm. There were no displacements or complications. We conclude that in selected patients with tachyarrhythmias, ambulatory monitoring of the atrial electrogram is a safe and useful addition to the technique of ECG monitoring offering an occasional therapeutic option.

\section{Ventricular tachycardia misdiagnosed as supraventricular tachycardia}

\section{Dancy, Linda Scaffidi, A J Camm, D Ward Cardiology Departments, St George's Hospital and St Bartholomew's Hospital, London}

The differential diagnosis of broad QRS complex tachycardias can be difficult and despite well defined criteria misdiagnosis of ventricular as supraventricular tachycardia with aberration is still common. Over a five month period in two institutions we have seen 21 instances of this problem. The history and 6212 lead ECGs during ventricular tachycardia (VT) were reviewed to see if strict application of existing criteria would have enabled earlier correct diagnosis. In 16/21 patients there was a past history of myocardial infarction, 9/21 had a documented LV aneurysm, and 10/21 had previous ECG evidence of complex ventricular ectopy. VT was haemodynamically stable in 14/21. In $10 / 21$ patients and 34\% of ECGs diagnostic features of VT were present (AV dissociation, fusions, captures). QRS width was $>140 \mathrm{~ms}$ in $58 \%$ of episodes. Left bundle branch block QRS type was seen in $18 \%$, right bundle branch block type in $31 \%$, and indeterminate in $51 \%$. $1: 1$ retrograde conduction was seen in $8 \%$, left axis deviation $\left(<-45^{\circ}\right)$ in $37 \%$, and precordial QRS "concordance" in $26 \%$. In $4 / 21$ patients two or more QRS types during separate episodes were noted. In $19 / 21$ verapamil was given intravenously on at least one occasion and never with success. In 17/21 patients intracardiac studies proved a ventricular origin. Careful analysis of ECGs could have resulted in correct diagnosis in most cases with consequent important influences on subsequent management. Other features which appeared to contribute to misdiagnosis included reluctance to make a diagnosis of VT, misconception that VT always causes circulatory col- 
lapse, failure to take into account past history, and unwillingness to change an established diagnosis. These patients highlight the importance of careful review of all data before VT is excluded and inappropriate management instituted.

\section{Death and damage after multiple DC countershocks}

Carol M Wilson, J D Allen, A A Jennifer Adgey

Cardiac and Physiology Department, Royal Victoria Hospital and Queen's University, Belfast, N Ireland

Many patients who receive multiple defibrillator shocks have a poor prognosis. Is this due to the underlying disease or to adverse effects of the shocks? Greyhounds $(25.4(0.79) \mathrm{kg})$, with a heart weight comparable to that of man, were anaesthetised with pentobarbitone $(30 \mathrm{mg} / \mathrm{kg}$ ) and received either 1,5 , or 10 synchronised transthoracic shocks of $400 \mathrm{~J}$ at intervals of $30 \mathrm{~s}$ from a Lown-type defibrillator. Acute mortality increased with increasing numbers of shocks (one shock $0 / 6$; five shocks $8 / 18 ; 10$ shocks 12/17: $\mathrm{p}<0.025)$. The terminal ECG showed unco-ordinated irregular atrial and ventricular activity, progressing within minutes to asystole. Electron and light microscopy at this time showed no reproducible lesions in the contractile apparatus. Three days after the shocks the hearts of five survivors in each group were excised. These were sliced and incubated in $1 \%$ triphenyltetrazolium chloride (TTC). The one shock group showed little damage $(0.004(0.004) \mathrm{G}$; mean (SEM)), few associated ECG changes, and minimal ventricular ectopic activity during the three day survival. The five shock $(7 \cdot 2(2 \cdot 0) \mathrm{G})$ and 10 shock groups $(13 \cdot 1(1.8)$ G) showed considerable damage with transmural right ventricular involvement, acute injury changes in the ECG and a late sustained ventricular tachycardia of the Harris type which was maximal at $\mathbf{2 4}$ hours following the shocks. Optimised first shock effectiveness is essential to minimise the clinical need for 5-10 shocks, associated here with gross cardiac damage and acute mortality.

\section{Haematological and tissue effects of high energy ablation}

E G C A Boyd, P Holt

Departments of Clinical Physics, Bioengineering, and Cardiology, Guy's Hospital, London

Endocardial ablation has been performed with cathe- ter electrodes on the His bundle or in the coronary sinus. Application of the technique to ventricular tachycardia (VT) foci could involve the use of electrodes in the left ventricle (LV). We have therefore investigated the tissue and haematological effects plus gas production when high energies are delivered via standard USCI bipolar catheter electrodes (BE). A flexible plastic bag filled with $300 \mathrm{ml}$ of fresh citrated blood was suspended in a tank of normal saline. The distal electrode of the USCI BE was immersed in the blood and $40 \times 100 \mathrm{~J}$ delivered. Gas produced was collected and analysed and the blood assessed for haemolysis. Tissue experiments were performed on the left ventricle from freshly killed pigs. Myocardial temperature and pressure changes were recorded at various depths during delivery of $400 \mathrm{~J}$ impulses to the endocardium. Forty $100 \mathrm{~J}$ shocks produced $0.5 \mathrm{ml}$ of gas. Analysis showed release of hydrogen and reduction of oxygen. There was haemolysis of $3 \%$ of haemoglobin. The pressure measured was approximately $20 \mathrm{psi}, 2.5 \mathrm{~cm}$ from the electrode at a depth of $0.5 \mathrm{~cm}$ from the endocardium, falling to $5 \mathrm{psi}$ on the epicardium. Corresponding temperature rises were $0.13^{\circ} \mathrm{C}$ and $0.03^{\circ} \mathrm{C}$ respectively.

The small quantity of free gas, slight haemolysis, and pressure and temperature changes do not suggest that it is unsafe to apply high energies to the left ventricle endocardium in man.

\section{Origin of late potentials defined by ECG signal averaging}

R W F Campbell, P Kertes, M Glabus, A Murray, C J Hilton

Department of Cardiology, Freeman Hospital, Newcastle upon Tyne

Late potentials obtained by signal averaging of surface ECGs are thought to reflect myocardial depolarisation outside the normal duration of the QRS. Their precise origin is, however, uncertain. We compared the signal averaged ECGs of 11 patients with direct recordings of epicardial and endocardial electrograms at surgery. Surgery was: coronary grafting in two patients, endocardial resection for arrhythmias in six patients, aneurysmectomy in three patients. The timing, duration, and morphology of electrograms from 53 epicardial and 36 endocardial sites were compared with the averaged surface ECG obtained before or during surgery. Late potentials were recorded in seven patients (five with arrhythmias, two without). In six of the seven patients the timing of epicardial electrogram fragmentation was closely correlated with the 
timing of surface late potentials. Late potentials were seen if three or more epicardial sites showed fragmentation ( $>100 \mathrm{~ms}$ ). The seventh patient has QRS widening between averaging and surgery. Surgical excision of sites of fragmentation abolished late potentials. The four patients without late potentials (two with arrhythmias) showed no epicardial fragmentation. The relationship between endocardial fragmentation and late potentials was less clear. In three patients, the duration of endocardial fragmentation (more than three sites) exceeded that of epicardial fragmentation but was not reflected as late potentials.

We conclude that late potentials represent fragmented delayed ventricular activation occurring predominantly in the epicardial myocardium.

\section{Physiological pacing in children}

J V de Giovanni, E D Silove

Department of Cardiology, Birmingham Children's Hospital, Birmingham

Active children with symptomatic heart block require pacing at constantly high rates to allow for the variable demand. Physiological pacing, therefore, would appear ideally suited for this group of patients but there are specific technique problems. We implanted dual chamber pacemakers in four patients aged between 11 and 13 years. All had symptomatic complete heart block which was congenital in two and followed surgical repair for congenital heart disease in the other two. The technique differs from implantation in adults in allowing lead length for growth. The association of congenital heart disease may enlarge the right atrium and encourage early lead displacement, which occurred in two of our patients. Associated systemic venous anomalies may limit access to endocardial pacing. Two of our patients had previous demand units and have observed significant improvement in activity with the physiological system. The other two, paced for the first time, have also noticed other benefits in addition to the cessation of syncopal attacks. Two patients required reprogramming, one initially because of exercise induced angina and later for pacemaker mediated re-entry tachycardia.

Physiological pacing in children appears to produce significant benefits particularly during exercise, predominantly due to the rate response and perhaps to the contribution from atrial transport.
His bundle ablation using lower energies and an active fixation electrode

P Holt, E Boyd, J C P Crick, E Sowton

Departments of Cardiology and Clinical Physics and

Bioengineering, Guy's Hospital, London

Endocardial ablation of the His bundle has previously been attempted using high energies and standard catheter electrodes. We have attempted to reduce energy requirements by use of an active fixation electrode. Five patients (four men, one woman) aged 48-69 years were studied. Four had paroxysmal atrial fibrillation (PAF) and one had tachycardias due to dual AV nodal pathways (DAVN). Pre-ablation, AV node Wenckebach occurred at $150-220$ beats/min (mean 176/minute), VA Wenckebach 160-200 beats/minute (mean 172/minute). A temporary pacing electrode was inserted into the right ventricle, and a Vitatron Helifix (VH) electrode introduced via the right femoral vein and positioned to obtain the maximal His bundle deflection. Five shocks $(1 \times 25$ and $4 \times 50 \mathrm{~J}$ ) were delivered via the $\mathrm{VH}$ at one minute intervals. The other output of the defibrillator was connected to a paddle placed on the posterior chest wall. Blood for cardiac enzyme estimation was then obtained. All patients developed complete heart block (CHB) immediately postablation; the two patients in atrial fibrillation reverted to regular sinus node activity. Complete heart block persisted in two patients and was intermittent in one. All required permanent pacemakers. Two patients had first degree AV block and right bundle branch block one month postablation (mean AV Wenckebach 120/minute, VA 100/ minute). All patients remained asymptomatic. Maximum creatine phosphokinase values occurred one hour postablation with no significant rise in creatine phosphokinase MB.

Therefore His bundle ablation can be safely and successfully performed using an active fixation electrode and lower energies. This method may prove valuable for ablation of other cardiac sites involved in tachyarrhythmias.

Effect of antiarrhythmic drugs and atrial pacing on atrial repolarisation using high gain surface electrocardiography

Nadia M G Debbas, A W Nathan, T Cochrane, A M Levy, G S Butrous, A J Camm

Department of Cardiology, St Bartholomew's Hospital, London

High fidelity (0.16 to $30 \mathrm{~Hz})$, high gain (x 1000 to $x 10$ 
000) ECGs (leads I, II, and III) were recorded from 29 supine patients with heart block. The $\mathrm{P}$ wave duration (atrial depolarisation), the length of $\mathrm{Ta}$ wave (atrial repolarisation), and the total interval were measured from five non-conducted atrial events. Progressively increasing the atrial pacing rate from 80 to 150 beats/min resulted in a linear reduction of the PTa interval from 430 to $280 \mathrm{~ms}$ ( $\mathrm{rho}=0.66$, slope $=0.29$ ). This was due largely to shortening of the Ta component. Atropine $(0.02 \mathrm{mg} / \mathrm{kg})$ shortened the PP interval (748(96) to 581(69) ms, $\mathrm{p}<0.001)$ and the PTa interval $(386(29)$ to $355(99) \mathrm{ms}, \mathrm{p}<0.01)$. Propranolol $(0.15 \mathrm{mg} / \mathrm{kg})$ prolonged the PP interval $(699(82)$ to $826(99) \mathrm{ms}, \mathrm{p}<0.001$ ) but did not alter the PTa interval (405(19) to $405(37) \mathrm{ms}$, NS). Neither disopyramide $(2.0 \mathrm{mg} / \mathrm{kg})$ nor flecainide $(2.0 \mathrm{mg} / \mathrm{kg})$ altered the PP interval (732(77) to 678(68) $\mathrm{ms}(\mathrm{NS})$ for disopyramide and $724(91)$ to $704(52) \mathrm{ms}$ (NS) for flecainide) but both prolonged the PTa interval (394(14) to 429(13) ms, $\mathrm{p}<0.001$ for disopyramide and $400(20)$ to $433(11) \mathrm{ms}, \mathrm{p}<0.001$ for flecainide). The increase in PTa after flecainide was largely due to $P$ wave lengthening (P: $104(11)$ to $129(18) \mathrm{ms}$, $\mathrm{p}<0.001$; Ta: 296(19) to 304(19) ms, NS) whereas after disopyramide most of the PTa increase was due to Ta changes (P: 102(8) to $109(7) \mathrm{ms}$, NS; Ta: 292(10) to $320(14) \mathrm{ms}, \mathrm{p}<0.001)$. Atropine shortened both components of the PTa (P: 102(12) to $95(12) \mathrm{ms}$, $\mathrm{p}<0.001$; Ta: $284(22)$ to $260(31) \mathrm{ms}, \mathrm{p}<0.001)$ and propranolol had no effect (P: 104(5) to $105(7) \mathrm{ms}$, NS; Ta: $301(20)$ to $300(39) \mathrm{ms}, \mathrm{NS}$ ).

In heart block both $\mathrm{P}$ and $\mathrm{Ta}$ waves can be reliably measured and the effects of pacing and drugs on the atrial myocardium can be assessed. The results are consistent with cellular electrophysiology and similar to the effects on the human ventricle.

Quantitative comparative analysis of argon and Nd-YAG laser radiation of normal and atheromatous arterial walls

T J Bowker, S G Bown, P A Poole-Wilson, K M Fox, $P$ R Salmon, A F Rickards

National Heart Hospital and University College

Hospital, London

In some cases submitted for percutaneous transluminal balloon angioplasty the stenosis is too narrow to be traversed with the balloon or guide wire or both. An alternative approach is to use fibre-optically guided laser radiation to vaporise transluminally some of the occluding atheroma. The continuous wave Nd-YAG and argon lasers employ optical fibres and are used clinically. We have quantified the effects of these two lasers on normal and atheromatous arterial wall. Three hundred and fifty five separate craters were made in samples of normal and atheromatous human postmortem aortic wall by pulse durations from 0.1 to $5.0 \mathrm{~s}$ of Nd-YAG and argon radiation, of power ranges 5 to $70 \mathrm{~W}$ and 1 to $3 \mathrm{~W}$ respectively. Crater depths were measured to the nearest $0.05 \mathrm{~mm}$. For both lasers, crater depth was proportional to pulse energy rather than power. With Nd-YAG radiation, to maintain crater depths within $0.8 \mathrm{~mm}$ the maximum permissible pulse energy was $12 \mathrm{~J}$ for normal and $8 \mathrm{~J}$ for atheromatous aortic wall. For smaller crater depths, similar differences in maximum pulse energies were noted between normal and atheromatous aortic wall. Argon radiation did not show similar selectivity for atheromatous aortic wall, the corresponding maximum pulse energies being $1 \mathrm{~J}$ for $1 \mathrm{~mm}$ crater depth, $0.6 \mathrm{~J}$ for $0.6 \mathrm{~mm}$, and $0.2 \mathrm{~J}$ for $0.4 \mathrm{~mm}$ for both normal and atheromatous aortic wall. Percutaneous transluminal delivery of laser energy to the coronary circulation will require optical fibre core diameters of $200 \mu \mathrm{m}$ or less. With the argon laser a $200 \mu \mathrm{m}$ core fibre was used. Nevertheless, the optics of the Nd-YAG laser-fibre interface were such that to transmit power sufficient to produce a predictable effect on the target, a fibre core diameter of at least $400 \mu \mathrm{m}$ was necessary. Thus although the Nd-YAG's selective effect upon atheroma makes it theoretically preferable to the argon, in practice the optics of the system dictate that the argon laser is likely to be the more appropriate for coronary angioplasty.

\section{Differential transmission spectrometry of normal and atheromatous arterial wall}

A F Rickards, T J Bowker, P Edwards, T A Hall, M Regel, S G Bown, K M Fox, P A Poole-Wilson National Heart Hospital and University College Hospital, London, and University of Essex

For the technique of laser angioplasty to be safe and reliable, the laser energy must not only be direct at, but also absorbed by, atheromatous plaque rather than normal arterial wall. For any given wavelength, the degree of light absorption (and hence vaporisation) which takes place is an inherent physical property of the target; and it is important to know this property (optical absorption) for both atheroma and normal arterial wall. We measured the optical transmission from $450 \mathrm{~nm}$ to $800 \mathrm{~nm}$ of five samples each of atheromatous and of normal human postmortem 
aortic wall. With emitting and receiving optical fibres on either side of the sample, chopped white light was passed through the sample into a monochrometer to select wavelengths from $450 \mathrm{~nm}$ to $800 \mathrm{~nm}$, whose intensities were measured by a phase sensitive detector. The distance between the emitting and receiving optical fibre tipes could be adjusted and measured by a micromanipulator. In both normal and atheromatous aortic wall the percentage optical transmission varied inversely with thickness of sample and directly with wavelength. For a thickness of $0.9 \mathrm{~mm}$, the optical transmission of normal and atheromatous aortic wall fell from $45 \%$ and $22 \%$ respectively at $750 \mathrm{~nm}$ to $12 \%$ and $2.5 \%$ respectively at $450 \mathrm{~nm}$. The ratio of the optical transmission per unit thickness of normal aortic wall to that of atheromatous aortic wall was 2:1 from $800 \mathrm{~nm}$ to $600 \mathrm{~nm}$, rising to $5: 1$ at $500 \mathrm{~nm}$.

Thus between $800 \mathrm{~nm}$ and $450 \mathrm{~nm}$ atheteromatous aortic wall absorbs light more strongly per unit thickness than normal aortic wall, and this effect is most pronounced at $500 \mathrm{~nm}$. Such wavelength-dependent differential optical absorption between normal and atheromatous aortic wall can be used to improve the selectivity and safety of laser angioplasty.

Apolipoprotein gene polymorphisms as markers for atherosclerosis

A Rees, N I Jowett, J L Caplin, J Stocks, L G Williams, M A Vella, F E Baralle, A J Camm, D J Galton

Departments of Medicine and Cardiology, St Bartholomew's Hospital, London

A recent report suggests that certain DNA polymorphisms may provide genetic markers for atherosclerosis. We have described a DNA polymorphism at the apolipoprotein A-I/C-III gene cluster, which associates with hypertriglyceridaemia. In view of the importance of these apolipoproteins in high density lipoprotein (HDL) and very low density lipoprotein (VLDL) metabolism, and the relationship between HDL levels and the development of atherosclerosis, we have studied the distribution of this polymorphism in groups of patients with or without angiographically defined coronary atherosclerosis. Leucocyte DNA from Caucasian subjects was digested with the restriction endonuclease Sst-I, Southern blotted and hybridised with a ${ }^{32} \mathrm{P}$-labelled M13 genomic clone containing sequences coding for apolipoprotein A-I. Restriction fragments hybridising to the apo A-I probe were detected by autoradiography. The polymorphism was revealed by the presence of either a $4.2 \mathrm{~Kb}$ or $3.2 \mathrm{~Kb}$ restriction fragment. The $3.2 \mathrm{~Kb}$ allele is found in $30 \%$ of subjects with hypertriglyceridaemia but in less than $5 \%$ of controls. The $3.2 \mathrm{~Kb}$ allele was found in five of 33 subjects with extensive coronary atherosclerosis, and in two of 34 subjects with normal coronary arteries. These subjects were age and sex matched. The difference in distribution is not statistically significant, yet the possession of the $3.2 \mathrm{~Kb}$ allele may still be implicated in the development of coronary atherosclerosis. Further studies are required using other lipoprotein gene polymorphisms to establish their relevance to the development of coronary atherosclerosis.

High density lipoprotein-cholesterol, alcohol intake and ischaemic heart disease

S J Pocock, A G Shaper, A N Phillips, M Walker Royal Free Hospital School of Medicine, London

High density lipoprotein-cholesterol (HDL-C) is regarded as a well established protective factor in ischaemic heart disease. Results from the prospective phase of the British Regional Heart Study, involving 7735 middle aged men, show that low levels of HDL-C are associated with increased risk of ischaemic heart disease but that there is no evidence of a protective effect from higher levels. A critical review of four other prospective studies of HDL-C and ischaemia heart disease suggests that the importance of HDL-C as a protective factor may have been exaggerated. HDL-C concentration is increased by alcohol consumption and this finding is used to support the widely held view that moderate drinking is protective against ischaemic heart disease. Results from the Regional Heart Study confirm the increase in HDL-C concentration with increasing alcohol intake but provide no evidence of any protective effect of alcohol on the risk of major ischaemic heart disease. Overall, results from this study suggest that HDL-C is neither a useful predictor of the risk of ischaemic heart disease nor an important protective factor in ischaemic heart disease in these middle aged British men. There is also no support for the view that moderate drinking may protect against ischaemic heart disease. 
Occult coronary artery disease in familial hypercholesterolaemia

W G Hendry, Mary Seed, A M Salmasi, J W Densem, R Niththyananthan

St Mary's Hospital, London

Familial hypercholesterolaemia (FH) is well know as a risk factor for coronary artery disease. We have investigated the presence of coronary artery disease in $\mathbf{6 0}$ asymptomatic patients ( 19 female, 41 male), mean age 40.5 years with $\mathrm{FH}$ and a normal resting ECG. FH was diagnosed on standard criteria: fasting total cholesterol $>7.3 \mathrm{mmol} / \mathrm{l}$ and low density lipoprotein $>4.4 \mathrm{mmol} / \mathrm{l}$, a family history of early arterial disease in a first degree relative, and/or presence of xanthelasma, early corneal arcus, or tendon xanthoma. Other major risk factors for coronary artery disease (diabetes, smoking, hypertension, and obesity) were present with expected frequency relative to the general population or were under-represented. Patients were investigated by 16 lead chest wall mapping during and after exercise. Thirty patients $(50 \%)$ had positive tests, and, of these, 19 underwent coronary angiography. Significant narrowing $(>50 \%)$ in one or more vessels was present in 18. During four years of follow up coronary events occurred in 16 of the 30: 14 had angina with 11 proceeding to coronary artery bypass grafting and two had a myocardial infarct. There were no such events in the 30 patients with negative exercise tests. This group had a lower total cholesterol: $7 \cdot 9(2 \cdot 1) \mathrm{mmol} / \mathrm{l}$ (mean (SD)) vs 9.3(1.6) $\mathrm{mmol} / \mathrm{l}(\mathrm{p}<0.01)$ and a higher density lipoprotein: $1.29(0.23) \mathrm{mmol} / \mathrm{l}$ vs $1.5(0.25) \mathrm{mmol} / \mathrm{l}(\mathrm{p}<0.05)$, but showed no difference in any other risk factor when compared with the 30 patients with positive exercise tests. This study demonstrates the ability of the exercise test to detect occult coronary artery disease and to predict subsequent major coronary events in patients with FH. The increased risk appears to be related solely to the level of the lipoprotein abnormality.

\section{Underestimation of coronary arterial stenosis by arteriography}

N Dilly, A C Thomas, F Franc, M J Davies

St George's Hospital Medical School, London, and Institute of Physics, Czechoslovakian Academy of Science, Prague, Czechoslovakia

Postmortem perfusion-fixation of coronary arteries at physiological pressures has shown that slit-like and crescentic lumens do not occur with uncomplicated atheromatous plaques. The residual lumen with plaques uncomplicated by acute thrombosis is usually eccentric in position but may be circular, ovoid, or D-shaped. Using a computer program we have shown there is a significant variation of arterial stenosis in different planes with ovoid or D-shaped lumens. The optimum shape which might be totally missed by examination in one radiological plane is not a cresent but an ovoid, the long axis of which is the same length as the normal vessel diameter. To prove this experimentally we have used glass tubes with artificially created stenoses in which the residual lumen is ovoid to stimulate diseased coronary arteries. $X$ rays of these barium filled glass tubes through different degrees of rotation show that the percentage stenosis by diameter may vary by as much as $\mathbf{4 2}$ depending on the plane of view. Similarly underestimation may be seen if the incident $x$ ray is not perpendicular to the object.

It is no longer necessary to suggest the occurrence of slit-like and crescentic lumens in order to explain underestimation of coronary stenosis in clinical coronary arteriography.

\section{The crescentic lumen-fact or fiction?}

A C Thomas, M J Davies, N Dilly, F Franc St George's Hospital Medical School, London, and Institute of Physics, Czechoslovakian Academy of Science, Prague, Czechoslovakia

It is widely accepted that some significant stenoses can be seen only in one plane at coronary arteriography. The explanation of this phenomenon is usually taken to be the slit-like or crescentic shape of the coronary arterial lumen. Numerous illustrations of such luminal shapes are published but derive from arteries fixed for pathological examination in the collapsed state. Coronary arteries fixed after distention at physiological pressures give a far better approximation to the shape of the lumen in life. Hearts have been perfused fixed with formol saline at pressures of 100 and 150 $\mathrm{mm} \mathrm{Hg}$ by tying a large cannula in the aorta. At such a pressure the aortic valve closes and the coronary arteries are perfused. The specimen is perfused for 24 hours after which fixation has produced enough rigidity for the vessels to remain in the fully distended state. The arteries are subsequently dissected en bloc free from the heart, decalcified, and examined by transverse cuts at $2 \mathrm{~mm}$ intervals. Normal arterial segments have a completely circular lumen. The 
internal elastic lamina is straight suggesting the artery is in the state analogous to complete dilatation. In diseased segments the majority of the lumens approximate to being circular although often placed eccentric to the mid-line of the vessel. Atheromatous plaques bulge outward at the expense of the media not inward to project into the lumen. Ovoid and D-shaped lumens are seen and are caused by flattening of the cap of hard fibrous plaques. Slit-like and crescentic lumens only occur with acute evolving lesions where a polypoidal mass of thrombus projects into the lumen.

\section{Prevalence, diagnosis and prognosis of right ventricular necrosis in acute inferior myocardial infarction}

N G Dewhurst, E Rodrigues, Lesley M Smart, W J Hannan, A L Muir

Departments of Medicine, Cardiology, and Medical Physics, Royal Infirmary, Edinburgh

To compare the non-invasive methods of diagnosis of right ventricular necrosis, 51 consecutive patients with inferior myocardial infarction underwent serial radionuclide ventriculography, pyrophosphate (PYP) scintigraphy, cross sectional echocardiography, and 12 lead electrocardiography with the addition of unipolar right chest lead V4R. All 51 scintigrams confirmed inferior infarction, and additional uptake in the right ventricle was noted in 25. Significant correlation was observed between right ventricular ejection fraction (RVEF) determined by bolus and gated radionuclide techniques $(r=0.92)$ and although an echocardiographic estimate was achieved in $94 \%$ of patients all possible views were obtained in only $63 \%$ and agreement with the radioisotope methods was poor $(r=0.45)$. Clinical recognition of right ventricular infarction was noted in only three patients (mean RVEF 0.16) but severe right ventricular dysfunction (RVEF $<0.25$ ) was seen in a further 22, and of these six suffered late hospital deaths. ST segment elevation $\geqslant 0.5 \mathrm{~mm}$ in V4R had a sensitivity of $92 \%$ in the recognition of severe right ventricular dysfunction but had a low specificity (40\%). Those surviving hospital stay failed to show any improvement in resting RVEF two months later, six exhibiting persisting right ventricular dyskinesis and three developing features of predominant right ventricular failure.

Clinical recognition of right ventricular infarction was poor, use of the right chest lead V4R could lead to overdiagnosis, and echocardiography was limited by the complex geometry of the right ventricle. Three patients with poor right ventricular function had no evidence of right ventricular uptake by the PYP scintigram. We believe that functionally important right ventricular infarction is best detected by radionuclide ventriculography, particularly as profound right ventricular dysfunction appears to carry a high late hospital mortality.

\section{Left ventricular thrombus in acute myocardial infarction: recognition by cross sectional echocardiography}

\section{R A Foale, P Nihoyannopoulos, Gillian Smith, A Maseri \\ Cardiovascular Unit, Royal Postgraduate Medical School, Hammersmith Hospital, London}

Anticoagulants have been advocated in acute myocardial infarction, when left ventricular thrombus is recognised by echocardiography. Of $M$ mode and cross sectional echocardiographic studies in 95 consecutive patients with first transmural myocardial infarction and no anticoagulant therapy, 72 were suitable for serial evaluation of left ventricular thrombus. Echocardiography findings were related to patients' outcome. Thirty two patients had inferior myocardial infarction, and none had left ventricular thrombus. Peak creatine phosphokinase (CPK) values was 1633(1244) IU (mean (SD)). There was 1/32 deaths. Forty patients had anterior myocardial infarction. Sixteen of 40 (40\%) had left ventricular thrombus on one or more echocardiographic studies (group 1). Twenty four patients with anterior myocardial infarction had no left ventricular thrombus (group 2). There was no difference in myocardial infarct size in group 1 compared with group 2 as judged by CPK values (2440(1411) vs 2271(1257) IU). In group 1, left ventricular thrombus appeared variously at three hours to 10 days postinfarction and in three patients spontaneously resolved. The presence of left ventricular thrombus was not unfavourably related to survival and functional class post myocardial infarction. There was 1/16 deaths and a mean class 1b (NYHA classification) in group 1 compared with $12 / 24$ deaths $(p<0.05)$ and mean class $2 b(p<0.01)$ in group 2 . Two deaths in group 2 were due to cardiac rupture. Systemic embolism occurred in one patient only who had inferior myocardial infarction and no left ventricular thrombus by echocardiography. Left ventricular thrombus is not predictable and is subject to spontaneous lysis. The presence of left ventricular thrombus detected by cross sectional echocardiography may not be associated with an unfavourable risk and, by splinting the infarcted segment, may even be beneficial following anterior myocardial infarction. 
Reciprocal ST depression in acute myocardial infarction: relationship to coronary artery disease

O Odemuyiwa, I Peart, Catherine Albers, R J C Hall Royal Victoria Infirmary, Newcastle upon Tyne

ST segment elevation in leads remote from those showing ST elevation during acute myocardial infarction (MI) has been attributed to benign electrical phenomena, distant myocardial ischaemia, or extensive myocardial damage. We studied 84 consecutive patients under 55 years with a first transmural MI. Treadmill exercise tests were performed at three and six weeks postinfarction. All had coronary angiography. Twenty eight $(75 \%)$ of the 51 inferior and 19 (58\%) of the 33 anterior infarcts had reciprocal ST depression of $\geqslant 1 \mathrm{~mm}$ during the acute phase. Fourteen (38\%) of the 38 inferior infarcts with reciprocal depression and $3(23 \%)$ of the 13 without reciprocal depression had significant left anterior descending (LAD) disease (NS). In inferior infarction reciprocal depression does not therefore predict LAD disease. Ten $(26 \%)$ of the $3 \delta$ inferior infarcts with reciprocal depression had ST depression in the same leads on exercise and five (50\%) of these patients had significant LAD disease. Nine $(32 \%)$ of the 28 patients who did not have ST depression in the same leads on exercise had significant LAD disease (NS). Seven (37\%) of the 19 anterior infarcts with reciprocal depression and four (29\%) of the 14 without reciprocal depression had significant right coronary artery (RCA) disease (NS). Reciprocal depression in anterior infarcts therefore does not predict significant RCA disease. Only one patient with anterior infarction had ST depression in the same leads on exercise and there was no significant RCA disease.

In conclusion, neither reciprocal ST depression during acute myocardial infarction nor exercise induced ST depression in the same leads early post infarction predict significant coronary artery disease in the reciprocal territory.

Digital subtraction angiography: a screening procedure for patency of coronary artery bypass grafts

G Hunter, S Walton, R Hayward, R H Swanton, $R$ Emanuel

Departments of Imaging and Cardiology, Middlesex Hospital, London

A prospective study was undertaken to evaluate ven- ous digital subtraction angiography (DSA) in the detection of coronary graft presence and patency. Patients with recurrence of chest pain following coronary grafting were studied by venous DSA and conventional angiography. Forty millilitres of iohexol were injected into the right atrium via a $5 \mathrm{~F}$ catheter at $25 \mathrm{ml}$ per second per run. Images were acquired in both left anterior oblique $45^{\circ}$ and right anterior oblique $5^{\circ}$ projections. Graft patency and presence was assessed by three independent observers without prior knowledge of which grafts had been inserted. Sixteen patients with 37 grafts were studied. Twenty six grafts were patent. There was agreement in 11 patients, seven being correctly assessed. There was partial agreement in the remaining five patients. All occluded grafts were correctly identified; 17,18 , and 19 of the 26 patent grafts were identified by the three observers respectively. The graft to the left anterior descending artery was least well demonstrated, with five out of 12 patent grafts missed. Patent grafts to the left circumflex and posterior descending arteries were not identified in one out of six and two out of eight cases respectively.

These figures yield a specificity of $100 \%$ (no false positives) and a sensitivity of $78 \%$. A graft identified by DSA is definitely present but patient grafts may be missed.

Free radicals and ischaemic injury: allopurinol improves myocardial protection during global ischaemia

D J Chambers, $M$ V Braimbridge, D J Hearse Heart Research Unit, Rayne Institute, St Thomas' Hospital, London

Ischaemia-induced breakdown of adenine nucleotides provides increased substrate for xanthine oxidase (the enzyme responsible for uric acid production from hypoxanthine and xanthine). Subsequent aerobic reperfusion increases oxygen availability so that xanthine oxidase activity and the consequent production of the superoxide free radical increases dramatically. During ischaemia and particularly during reperfusion, superoxide free radicals might play a role in cellular injury. We have investigated whether pretreatment with the specific xanthine oxidase inhibitor, allopurinol, can enhance myocardial resistance to ischaemia and reperfusion injury during normothermic $\left(37^{\circ} \mathrm{C}\right)$ and hypothermic $\left(21^{\circ} \mathrm{C}\right)$ arrest with St Thomas' Hospital cardioplegic solution. Rats were pretreated with allopurinol $(20 \mathrm{mg} / \mathrm{kg}$ orally for two 
days and intravenously on day 3 prior to excision of the heart). Using an isolated "working" rat heart preparation, hearts were perfused aerobically for 20 minutes followed by three minutes of cardioplegic infusion and either 27 minutes of $37^{\circ} \mathrm{C}$ global ischaemia or 300 minutes of $21^{\circ} \mathrm{C}$ global ischaemia (with three minute reinfusions of cardioplegia every 30 minutes) and reperfusion for 15 minutes. Postischaemic recovery (expressed as a percentage of preischaemic control) of aortic flow, cardiac output, stroke volume, and stroke work in the $37^{\circ} \mathrm{C}$ control group $(\mathbf{n}=8)$ were $22 \cdot 1(4.5) \%, \quad 36.9(7.5) \%$, $40.9(9.7) \%$, and $36.4(11.0) \%$ respectively, whereas these values in the allopurinol pretreated group $(\mathrm{n}=8)$ were $54.8(16.3) \%, 61.6(15.1) \%, 59.2(16.1) \%$ and $51 \cdot 8(17 \cdot 2) \%$. Postischaemic recovery values for these indices in the $21^{\circ} \mathrm{C}$ control group $(n=6)$ were $21.0(11.0) \%, 29.2(9.4) \%, 30.8(9.3) \%$, and $24.8(9.1) \%$ and in the allopurinol pretreatment group $(n=6)$ they were $24.8(8 \cdot 1) \%, \quad 32 \cdot 2(8 \cdot 5) \%, \quad 37 \cdot 2(8 \cdot 1) \%$, and $31 \cdot 2(8 \cdot 5) \%$. Thus although allopurinol pretreatment significantly improved myocardial resistance to ischaemic injury at normothermia, there was no effect with hypothermia. The most likely explanation of these results is that both allopurinol and hypothermia act to inhibit superoxide free radical production but hypothermia masks any allopurinol effect.

Limitation of infarct size: a comparative study with six drugs

D M Yellon, D J Hearse, J M Downey

Heart Research Unit, Rayne Institute, St Thomas'

Hospital, London, and the Department of Physiology, University of South Alabama, USA

Using a closed chest dog, three calcium antagonists verapamil, nifedipine, and diltiazem, two beta blocking agents metoprolol and cetamolol, an antiinflammatory agent flurbiprofen, and a xanthine oxidase inhibitor allopurinol were evaluated for their ability to limit myocardial infarct size following either 24 or 48 hour periods of coronary artery embolisation. Coronary arteries were embolised by injecting $2.5 \mathrm{~mm}$ beads into the coronary network via a carotid artery cannula. Cerium-141 labelled microspheres were given immediately thereafter to mark the blood flow pattern around the ischaemic area. Continuous infusion of the drug for $\mathbf{2 4}$ or $\mathbf{4 8}$ hours (saline given to controls) was then initiated. At the end of the ischaemic time period the hearts were removed, sliced, and the infarct area visualised using tetrazolium staining techniques. The risk zone was vis- ualised following autoradiography of the slices. The results of these studies clearly show that both the beta blockers, metoprolol and cetamolol, as well as the non-steroidal anti-inflammatory agent flurbiprofen, had no effect in limiting infarct size; whereas the three calcium antagonists verapamil (44\% limitation at 24 hours), nifedipine (40\% limitation at 24 hours and $25 \%$ limitation at 48 hours), and diltiazem (30\% limitation at 48 hours) and the xanthine oxidase inhibitor allopurinol (42\% limitation at 24 hours), showed a significant $(p<0.001)$ effect on limiting the extent of tissue necrosis.

Thus it can be seen that only certain classes of pharmacological agents appear to limit myocardial infarct size in this experimental preparation. The mechanism of action of these that appear beneficial is at present being studied.

Untreated chronic heart failure: effects at rest and on exercise of introducing diuretic therapy

\author{
J Bayliss, M Norell, R Canepa-Anson, C Reid, \\ P Poole-Wilson \\ National Heart Hospital, London
}

The physiological effects of introducing diuretic therapy in patients with untreated chronic heart failure are poorly documented. We observed clinical and neuroendocrine variables, at rest and on upright exercise, before and after the introduction of frusemide in 10 patients with chronic heart failure who had been symptomatic for at least one month but who had not had any prior therapy. Five patients had dilated cardiomyopathy, four chronic ischaemic heart disease, and one rheumatic mitral valve disease. Before diuretics, the patients were breathless on mild exertion, had radiological cardiomegaly and pulmonary venous hypertension, and raised venous pressure or a fourth heart sound; one had peripheral oedema. Treadmill exercise time was $10.8(2.5)$ (mean (SEM)) minutes, using a slow protocol. Plasma renin activity and aldosterone were normal in all patients, at rest and on exercise. Plasma noradrenaline was elevated at rest and increased further on exercise. $(p<0.05)$. After one month of frusemide, patients were less breathless, had lost $3.6(1) \mathrm{kg}(\mathrm{p}<0.05)$, and doubled their exercise time. Plasma renin activity and aldosterone were now abnormally elevated at rest, and more so on exercise; plasma noradrenaline was normal at rest but remained elevated on exercise.

Although diuretics cause short term clinical improvement in heart failure, reducing resting sympathetic activity, they achieve this at the expense of 
activation of the renin-angiotensin system, which may have important long term implications for therapy and progression of the disease.

\section{Intravenous captopril for severe heart failure}

\author{
M Rademaker, T R D Shaw, C R W Edwards \\ Department of Medicine, Western General Hospital, \\ Edinburgh
}

We studied the effect of intravenous administration of captopril in 26 patients with chronic heart failure. Plasma free captopril was measured by radioimmunoassay. A bolus dose of $25 \mathrm{mg}$ (14 patients) produced a very rapid reduction in systemic vascular resistance $(-31 \%)$ with maximal change at $5 \mathrm{~min}$ after injection. Cardiac output rose $20 \%$ and mean systemic pressure fell $18 \%$. Pulmonary end diastolic and right atrial pressures fell more slowly with a nadir at 60 min. The effect on systemic vascular resistance decreased over $90 \mathrm{~min}$, when a $9 \%$ reduction persisted. There was an initial fast distribution phase of plasma free captopril while the elimination half life between 1.5 and $8 \mathrm{~h}$ after the dose was 3.36(SE $0.25) \mathrm{h}$. A series of seven incremental doses $(0.3125$ $\mathrm{mg}$ to $20 \mathrm{mg}$ total) was given to 12 patients. Progressive changes in systemic vascular resistance, cardiac output, and systemic pressure were seen between $0.3125-5 \mathrm{mg}$ with no further change thereafter. Plasma free captopril at the onset of the plateau of the dose-response curve was $150 \mathrm{ng} / \mathrm{ml}$ : this is considerably higher than predose concentrations found during chronic therapy with $25-50 \mathrm{mg}$ thrice daily and suggests that larger doses or more frequent administration might be needed for a sustained maximal effect.

Intravenous captopril will be useful for intensive care patients with cardiac failure.

\section{Renal vascular response to neuroendocrine vasodilatation in chronic heart failure}

\author{
J Bayliss, $M$ Norell, R Canepa-Anson, \\ P Poole-Wilson, G C Sutton \\ Hillingdon Hospital, Uxbridge, and National Heart \\ Hospital, London
}

Activation of the renin-angiotensin and sympathetic systems in chronic heart failure causes important renal vasoconstriction. The renal vascular response to vasodilatation by inhibition of these neuroendocrine systems was assessed in 15 patients with stable heart failure on diuretics: measuring renal plasma flow (with ${ }^{123} \mathrm{I}-\mathrm{OIH}$ ) and cardiac haemodynamics, to derive renal blood flow (RBF), renal vascular resistance, and the percentage cardiac output to the kidneys (\%CO), before and after one month each of captopril and prazosin, in a double blind crossover study. Captopril reduced systemic $(p<0.05)$ and renal $(\mathrm{p}<0.01)$ vascular resistance, increasing $\mathrm{RBF}$ $(p<0.05)$ and \%CO $(p<0.05)$. The increase in RBF correlated with control plasma renin activity $(r=0.53$, $\mathbf{p}<0.05$ ). Although prazosin also reduced systemic vascular resistance $(p<0.05)$, renal vascular resistance increased $(\mathrm{p}<0.01)$, and RBF and \%CO fell $(\mathrm{p}<0.05)$. Weight fell $1.7 \mathrm{~kg}(\mathrm{p}<0.05)$ on captopril but increased $3.0 \mathrm{~kg}(\mathrm{p}<0.05)$ on prazosin; these changes correlated inversely with $\mathrm{RBF}$ changes $(\mathrm{r}=-0.53, \mathrm{p}<0.01)$.

Sympathetic inhibition with prazosin causes systemic vasodilatation, which diverts blood from the kidney and may result in fluid retention. Inhibition of the renin-angiotensin system with captopril causes preferential renal vasodilatation and allows renal perfusion to improve.

Neuroendocrine and haemodynamic interactions during exercise in chronic heart failure: double blind comparison of captopril and prazosin
J Bayliss, $M$ Norell, $R$ Canepa-Anson, P Poole-Wilson, G C Sutton
Hillingdon Hospital, Uxbridge, and National Heart Hospital, London

We report the first double blind study of clinical, neuroendocrine, and haemodynamic interactions in moderate chronic heart failure, at rest and during upright exercise, comparing inhibition of the reninangiotensin-aldosterone (RAA) system (captopril) and of the alpha ${ }_{1}$ adrenergic sympathetic system (prazosin). Sixteen patients on diuretics were studied; each drug was crossed over at one month. Control plasma renin activity (PRA), aldosterone (Aldo), and noradrenaline (NA) were elevated at rest and increased on exercise $(p<0.05)$. Control left ventricular filling pressure (LVFP) was elevated, correlating with PRA and Aldo $(r>0.51, p<0.05)$. Systemic vascular resistance (SVR) was elevated, and vasodilatation during exercise was limited by PRA and NA release $(r=0.82$, $p<0.01 ; r=0.62, p<0.05)$. On captopril there were reductions in Aldo $(p<0.05)$ and LVFP $(p<0.05)$, at rest and on exercise, and dyspnoea was relieved. SVR was lower at rest $(p<0.05)$ and on exercise $(p<0.05)$; vasodilatation on exercise was no longer impaired by 
RAA activation, and exercise capacity increased by $35 \%(\mathrm{p}<0.01)$. On prazosin, NA and Aldo increased initially $(p<0.05)$, fluid retention increased weight $(p<0.05)$, LVFP was unchanged, and dyspnoea was not relieved. Resting SVR was reduced $(p<0.05)$, but exercise vasodilatation remained limited by RAA and sympathetic activation, and exercise capacity did not improve.

These results illustrate the importance of the renin-angiotensin-aldosterone system in chronic heart failure, at rest and also on exercise.

Right ventricular dysfunction dictates the therapeutic response in chronic cardiac failure?

Ann Tweddel, W Martin, I A McGhie, I Hutton University Department of Medical Cardiology and Department of Nuclear Medicine, Royal Infirmary, Glasgow

In patients with chronic cardiac failure, vasodilator therapy produces a variable therapeutic effect. The purpose of this study was to determine whether the response to afterload reduction is affected by associated right ventricular dysfunction in patients with chronic left ventricular failure. Fifteen male patients with New York Heart Association grade III-IV chronic cardiac failure secondary to coronary heart disease were studied. Heart rate was obtained from the electrocardiogram, blood pressure by cuff sphygmomanometer, cardiac output by thermodilution, and ejection fraction of right and left ventricle from gated technetium ventriculography. In 10 patients with preserved right ventricular function (RVEF $>25 \%$ ), LVEF ranged from 10 to $29 \%$. Left ventricular ejection fraction ranged from 10 to $23 \%$ in five patients with right ventricular dysfunction (RVEF $12-24 \%)$. Exercise tolerance was similar in both groups (mean exercise time 178(21) s and 146(16) s. In both groups afterload reduction with felodipine produced a modest increase in heart rate from $71(5)$ beats/min to $79(5)$ beats/min and $79(6)$ beats/min to 85 (7) beats/min secondary to fall in mean blood pressure from 101(4) $\mathrm{mm} \mathrm{Hg}$ to $87(4) \mathrm{mm} \mathrm{Hg}(p<0.05)$ and $83(5)$ to $78(5) \mathrm{mm} \mathrm{Hg}$. In patients with preserved right ventricular function cardiac index increased from 2.5 to $3.81 / \mathrm{min} / \mathrm{m}^{2}(p<0.01)$ but in patients with right ventricular dysfunction cardiac index increased insignificantly from $2.3(0.4)$ to $2.6(0.4) \mathrm{l} / \mathrm{min} / \mathrm{m}^{2}$. In conclusion, in patients with ischaemic chronic cardiac failure with a similar degree of left ventricular dysfunction, associated right ventricular abnormality predicts patients with a limited therapeutic response to afterload reduction.
Increased sensitivity of the denervated human heart to isoprenaline before and after beta blockade

S Yusuf, S Theodoropoulos, C J Mathias, N Dhalla, $M$ Yacoub

Harefield Hospital, Middlesex, and St Mary's Hospital, London

Denervation may increase target organ sensitivity to its chemical transmitter. We tested this hypothesis by studying the heart rate (HR) response to isoprenaline of the denervated donor heart (DH) as compared to the innervated recipient heart ( $\mathrm{RH})$ in eight patients who underwent heterotopic cardiac transplantation because of congestive cardiomyopathy (2) or ischaemic heart disease (6) six to 12 months earlier. All patients were well compensated and did not require treatment for heart failure. The resting heart rate was higher in the donor heart $(101.4(3.7)$ beats/ min compared to the recipient heart $(90.5(6.5)$ beats/min; $\mathrm{p}<0.04)$. Incremental intravenous infusion of isoprenaline $(5 \mathrm{ng}, 10 \mathrm{ng}$, and $15 \mathrm{ng} / \mathrm{kg} / \mathrm{min}$ ) raised heart rate to a greater extent in the donor heart at the two higher doses $(21.9(3.5)$ and $32.4(4.7)$ beats/min for donor heart $v s 14.8(2.7)$ and $22.8(4.2)$ beats/min for recipient heart; $p<0.01$ and $p<0.003$ respectively). Following $0.1 \mathrm{mg} / \mathrm{kg}$ of intravenous propranolol, the resting heart rate decreased similarly in the donor heart $(-12(2)$ beats $/ \mathrm{min} \mathrm{p}<0.01)$ and the recipient heart $(-14(2.5)$ beats $/ \mathrm{min} ; \mathrm{p}<0.01)$. There was little heart rate increase in both hearts at $5 \mathrm{ng}$ and $10 \mathrm{ng} / \mathrm{kg} / \mathrm{min}$, but at higher doses the donor heart was still more responsive $(15 \mathrm{ng} / \mathrm{kg} / \mathrm{min}: 11 \cdot 2(2 \cdot 1)$ beats/ min for donor heart vs $6.5(1.9)$ beats $/ \mathrm{min}$ for recipient heart; $\mathrm{p}<0.01 ; 20 \mathrm{ng} / \mathrm{kg} / \mathrm{min}: 14.1(2.8)$ beats $/ \mathrm{min}$ for donor heart vs $6.7(1.6)$ beats $/ \mathrm{min}$ for recipient heart; $\mathrm{p}<0.005 ; 25 \mathrm{ng} / \mathrm{kg} / \mathrm{min}: 17.7(3)$ beats $/ \mathrm{min}$ for donor heart vs $8 \cdot 1(1.6)$ beats/min for recipient heart; $\mathrm{p}<0.002$ ).

In patients with cardiac transplantation, the denervated heart is more sensitive to the chronotropic effect of isoprenaline. This may reflect increased density or affinity of the beta receptors in the denervated heart.

Beta blockade produces greater attenuation of exercise tachycardia in the denervated human heart

N Dhalla, S Yusuf, S Theodoropolous, C Reid, $M$ Yacoub

Harefield Hospital, Harefield, Middlesex; and St Mary's Hospital, London

Heterotopic cardiac transplantation offers an oppor- 
tunity to assess the effect of pharmacological agents on the innervated recipient and the denervated donor heart simultaneously in the same patient. The effect of acute beta blockade on the heart rate (HR) response to exercise was studied in six patients 6-12 months after heterotopic cardiac transplantation, using a modified Bruce protocol before and after the administration of intravenous propranolol $(0.1 \mathrm{mg} / \mathrm{kg})$. The resting heart rate decreased similarly and significantly $(\mathrm{p}<0.05)$ in both the donor (DHR 15.5(2)) and recipient heart $(14 \cdot 3(2 \cdot 6))$. Before beta blockade the heart rate response at peak exercise was similar for both the donor (DHR 38(3.5)) and recipient heart (RHR 48(3)). After beta blockade the peak exercise response for the donor heart was 20.9(3) compared to $38.3(3.5)$ for the recipient heart which represents a two fold greater attenuation in the donor heart. In contrast, the increase in heart rate of both donor and recipient hearts in early exercise (first three minutes of the Bruce protocol) as well as the decrease during the first three minutes post exercise was unaffected by beta blockade.

It is concluded that the heart rate response of the denervated heart, at peak exercise, is markedly blunted by beta blockade and that greater caution is required in using these drugs in transplanted patients.

\section{Adverse effect of high dose aspirin on prostacyclin generation by autogenous vein grafts}

A H Gershlick, D Syndercombe Court, A J Murday, C T Lewis, P G Mills

The London Hospital, London

External jugular vein grafting into the ipsilateral carotid artery in the rabbit was used as a model to study the effect of different doses of aspirin (ASA) given in combination with dipyridamole (DIP), or DIP alone on vein graft prostacyclin $\left(\mathrm{PGI}_{2}\right)$ production. Animal groups consisted of: $A$, no treatment $(\mathrm{n}=6): \mathrm{B}$, low dose ASA $(0.5 \mathrm{mg} / \mathrm{kg} / 24 \mathrm{~h})$ plus DIP $(\mathrm{n}=6): \mathrm{C}$, high dose ASA $(40 \mathrm{mg} / \mathrm{kg} / 24 \mathrm{~h})$ plus DIP $(n=6):$ D, DIP alone $(n=6)$. DIP $(8 \mathrm{mg} / \mathrm{kg} / 24 \mathrm{~h}$ in divided doses) was started $48 \mathrm{~h}$ prior to operation and ASA on the first postoperative day. Both drugs were given for one month. Vein grafts (VG) and nongrafted artery (A) and vein (V) were then removed, opened and placed in a template with the luminal surface forming the base of a well. Radioimmunoassay was used to measure PGI levels in $500 \mu \mathrm{l}$ aliquots of HEPES buffer placed in the well. Results from group A showed that grafting of a vein into the arterial system results in $\mathrm{PGI}_{2}$ levels which almost reached arter- ial levels (mean for $\mathrm{V}=122 \mathrm{pg}$ (range 104-144), $\mathrm{VG}=309 \mathrm{pg}$ (range 223-428), A=389 pg (range 299 507). Vein grafts and non-grafted vessels of group $C$ animals produced significantly less $\mathrm{PGI}_{2}$ than corresponding vessels of the other three groups $(p<0.01$ for all comparisons). In group $B$ animals there was a reduction in VG $\mathrm{PGI}_{2}$ as compared to group $\mathrm{A}$ animals, but the difference did not reach statistical significance.

High dose ASA therefore reduces prostacyclin in vein grafts, explaining the previous finding of enhanced platelet deposition in these animals. This in turn will cause the increase in graft intimal thickness also previously demonstrated. Because aspirin may have an adverse effect on long term graft survival through promotion of intimal hyperplasia, warfarin may be a better agent for the prevention of thrombotic occlusion in the early postoperative period.

Preparation of human saphenous vein for coronary surgery impairs prostacyclin production

\section{G Angelini, H Williams, J Psaila, A C Newby Departments of Cardiology, Cardiac Surgery and Surgery, University of Wales, Cardiff}

Immediate postoperative platelet deposition is thought to contribute both to early and to late coronary vein graft occlusion. We investigated whether routine preparation of saphenous vein leads to impaired prostacyclin production which would favour platelet deposition. Treatment-for example, with prostacyclin-might then offer scope for its prevention. Saphenous vein was harvested $(a)$ immediately after removal or $(b)$ after routine preparation by adventitial stripping, side branch ligation, $1 \mathrm{~min}$ distension at $<300 \mathrm{~mm} \mathrm{Hg}$, and storage for up to $2 \mathrm{~h}$ in $0.9 \mathrm{~g} / \mathrm{l} \mathrm{NaCl}$ at $23^{\circ} \mathrm{C}$. Weighed segments of vein were incubated for three $10 \mathrm{~min}$ periods in fresh $1 \mathrm{ml}$ aliquots of Tris buffer $(0.14 \mathrm{~mol} \mathrm{NaCl}, 15 \mathrm{mmol}$ Tris/ HCL, $\mathrm{pH}$ 7.4); each segment was then cut into three weighed pieces which were incubated for $3 \mathrm{~min}$ in $\mathbf{0 . 3}$ $\mathrm{ml}$ Tris buffer with vortex mixing for the first $15 \mathrm{~s}$. At the end of each incubation, the vein was removed, indomethacin $(3.3 \mu / \mathrm{ml})$ was added, and the 6-ketoprostaglandin $F_{1}$ concentration in the buffer was measured by radioimmunoassay. Unstimulated prostacyclin production was constant over the first three periods and averaged $1.7(0.5) \mathrm{pg} / \mathrm{ml} / \mathrm{min}$ in control vein and $1.9(0.5) \mathrm{pg} / \mathrm{ml} / \mathrm{min}$ in prepared vein (NS, $\mathrm{n}=5$ ). Prostacyclin production rose with vortex stimulation 11(2) fold in control vein but only 4(1) fold in prepared vein $(p<0.05)$. In a larger series $(n=13)$, 
vortex stimulated prostacyclin production proceeded at $16(2) \mathrm{pg} / \mathrm{mg} / \mathrm{min}$ in control vein and $7(1) \mathrm{pg} / \mathrm{mg} /$ min in prepared vein $(p<0.05)$. Morphological examination showed similar endothelial preservation in the segments from control and prepared vein. The data indicate that the capacity of vein to produce prostacyclin becomes impaired during surgical preparation.

Metabolic damage to human saphenous vein during preparation for coronary bypass surgery

A Newby, G Angelini

Departments of Cardiology and Cardiac Surgery, University of Wales, Cardiff

Coronary graft patency may be affected by the procedures used for intraoperative preparation of vein. Tissue damage caused during routine preparation and its component steps was quantified by measuring ATP and its metabolites by HPLC. Vein was frozen in liquid $\mathrm{N}_{2}$ after dissection (control); after storage in blood at $23^{\circ} \mathrm{C}$ for up to $2 \mathrm{~h}$ (stored); after side branch ligation and adventitial stripping (manipulated); after manipulation followed by $1 \mathrm{~min}$ distension at $<300$ $\mathrm{mm} \mathrm{Hg}$ (limited pressure) or with gentle unlimited pressure; or from vein unused after completion of the last proximal anastomosis (prepared vein). The ATP content of control vein, $480(30) \mathrm{nmol} / \mathrm{g}(\mathrm{n}=28)$, was reduced to $260(40) \mathrm{nmol} / \mathrm{g}(\mathrm{n}=15, \mathrm{p}<0.001)$ in prepared vein using distension at $<300 \mathrm{~mm} \mathrm{Hg}$; the ATP:ADP ratio was reduced from $2 \cdot 4(0 \cdot 1)$ to $1 \cdot 3(0 \cdot 1)$ $(p<0.001)$. The ATP content in vein prepared using unlimited pressure distension was $170(20) \mathrm{nmol} / \mathrm{g}$ $(n=23)$ and the ATP:ADP ratio was 0.9(0.1) $(p<0.05$ $v s$ distension at $<300 \mathrm{~mm} \mathrm{Hg}$ ). Inosine and hypoxanthine concentrations were elevated in prepared vein relative to control $(p<0.05)$. Storage caused a slow fall in ATP concentration to $350(40) \mathrm{nmol} / \mathrm{g}$ control $(n=6, p<0.05)$ but did not change ATP:ADP ratio. Manipulation alone caused no change in ATP content or ATP:ADP ratio, but manipulation followed by limited pressure distension reduced ATP:ADP ratio to $2 \cdot 1(0.1)(\mathrm{n}=8, \mathrm{p}<0.05$ vs control), and manipulation followed by unlimited pressure distension reduced ATP content to $250(20) \mathrm{nmol} / \mathrm{g}$ and ATP:ADP ratio to $1 \cdot 2(0 \cdot 1)(\mathrm{n}=8), \mathrm{p}<0.001)$. The data provide clear evidence of metabolic damage to vein during surgical preparation. The single step of unlimited pressure distension led to the same changes seen in prepared vein whereas storage was relatively innocuous.
Screening for carotid disease in patients with coronary artery disease

D A Ratliff, T K Hames, $\mathrm{K} N$ Humphries, N Conway, J Webster.

Wessex Regional Medical Physics and Cardiac Centres and Vascular Surgical Unit, Southampton

The prevalence of carotid disease has been variably reported as being present in up to $18 \%$ of patients with coronary artery disease (CAD) undergoing coronary artery bypass surgery. We have looked prospectively for evidence of carotid disease in 100 consecutive patients with symptomatic CAD undergoing coronary angiography and 100 controls having minor operations, using continuous wave (CW) Doppler imaging and Duplex scanning. CW Doppler imaging and Duplex scanning are accurate techniques in the direct non-invasive assessment of carotid disease. We have carried out a blind prospective comparison with biplanar angiography, in which the sensitivity and specificity of CW Doppler imaging was $90 \%$ and $98 \%$ respectively in the detection of $\geqslant 50 \%$ internal carotid stenosis $(n=96)$, and that of Duplex scanning was $93 \%$ and $98 \%$ respectively $(n=85)$. Ten per cent of CAD patients were found to have significant dișease $(\geqslant 50 \%$ stenosis or occlusion) affecting the internal carotid artery or carotid bifurcation compared with $3 \%$ of controls $(p<0.05)$. Seven CAD patients had no cerebral symptoms and five had no carotid bruit. Of nine affected patients who have had coronary angiography so far, four have undergone carotid endarterectomy prior to coronary artery bypass surgery without complication. A further two patients have been referred for carotid surgery.

Measurement of infarct size by thallium-201 rotating slant-hole tomography: a human pathological correlation

E Jane Flint, D N Taylor, J A Mills, J Pilcher, J A McIntosh

Departments of Cardiology and Clinical Physics, Walsgrave Hospital, Coventry

Thallium-201 scintigraphy was performed in 18 patients with a first myocardial infarct before death. All had rotating slant-hole tomography, 17 had anterior, $45^{\circ}$ left anterior oblique, and left lateral planar scans also. Post mortem examination was performed within four hours of death, hearts frozen, then sliced and incubated in nitroblue tetrazolium. Stereological analysis of heart slices to give percentage 
volume defect was compared with results from quantifying planar scans and 12 reconstructed tomographic slices. Three sets of tomograms and two planar series could not be assessed: movement artefact affected all scans in one patient; extensive apical defects in tomograms could not be quantified in two patients; unsuspected old infarction confused planar measurement in one patient, "cross talk" was observed in the tomograms. Blur artefacts were not a problem in this series but have been observed to hinder identification of smaller defects. Tomography improved the correlation of thallium defect size with pathology $(r=0.81)$ over planar scans $(r=0.65)$. Two patients had notably larger tomographic and planar thallium defects than pathological infarct size, presumably due to periinfarctive ischaemia. If these were excluded, tomographic infarct size correlated with pathological infarct size $(r=0.97)$, and for a subset of 11 patients with all data, tomography improved the correlation of thallium infarct size from $\mathrm{r}=0.88$ to $\mathrm{r}=\mathbf{0 . 9 5}$.

Thus thallium-201 rotating slant-hole tomography may be a useful research tool for sizing moderate first time infarcts in intervention studies, when satisfactory reconstruction can be achieved.

Clinical role of thallium-201 scintigraphy in the management and prognosis of coronary artery disease

M D Gammage, D P Murray, E Rafiqi, R G Murray Department of Cardiovascular Medicine, University of Birmingham and East Birmingham Hospital, Birmingham.

The clinical impact of thallium-201 (Tl-201) scintigraphy in coronary artery disease was reviewed in 128 consecutive patients who were referred for routine investigation and followed for a mean of 13(1) months. Exercise and redistribution image data were collected in multiple projections and interpreted by combined visual and semiquantitative means. A segmental image defect was accepted as indicating the presence of coronary artery disease. Normal Tl-201 images were obtained in 69 of $79(87 \%)$ diagnostic studies. Sixty four (82\%) were spared diagnostic coronary arteriography; five proceeded to coronary arteriography for persisting symptoms (two had normal arteriograms, two had minor ( $<70 \%$ stenosis) single vessel disease, one had double vessel disease). Conversely, coronary arteriography was normal in two of $10(20 \%)$ patients with unequivocal image defects. Tl-201 scintigraphy was performed as a func- tional complement to coronary arteriography in 49 patients, influencing the decision for surgery in 33 and determining the presence or absence of myocardial ischaemia in 16 patients with equivocal coronary arteriograms. Myocardial ischaemia was confirmed in eight of these 16 patients and normal data were obtained in eight patients. During the follow up period of 13(1) months none of the 77 patients with normal Tl-201 scintigrams died, sustained myocardial infarction, or other major cardiac event.

These data confirm both the vital role of Tl-201 scintigraphy in the management of patients with suspected or proven coronary artery disease and the excellent prognostic value of a normal Tl-201 scintigram.

A new technique for displaying tomographic images of perfused myocardium obtained using T1-201 and their relationship to the coronary arteries

C J Gibson, E E Laird, E D Williams, A Rajathurai, B Mittra

Regional Medical Physics Department, Dryburn Hospital, Durham; Sunderland District General Hospital; and Department of Medicine, Sunderland District General Hospital

Conventional transverse tomograms with thallium201 represent oblique slices through perfused myocardium which do not correspond to the major axes of the left ventricle. It is difficult to relate observed defects easily to coronary anatomy. We have developed a new technique for the presentation of myocardial tomograms which allows the observer to perceive the shape of the Tl-201 distribution directly. The surface of the myocardium is found by applying an interactive thresholding technique to a set of conventional transverse slices. Computer graphics techniques are used to display a "shaded image" of that surface on a TV screen, showing the three dimensional shape of the myocardial surface from any chosen aspect.

As a further aid to image interpretation we have digitised a set of normal preserved coronary arteries. Using scaling and transformation techniques these arteries are "mapped" on to the myocardial tomograms, and a shaded surface image produced with superimposed coronary arteries. This provides a familiar anatomical framework for locating perfusion defects and may be of value in the identification of diseased vessels. 
Improved detection of coronary heart disease by gated thallium scanning

Ann Tweddel, W Martin, I A McGhie, I Hutton University Department of Medical Cardiology, and Department of Nuclear Medicine, Royal Infirmary, Glasgow

Thallium 201 imaging is established for the assessment of myocardial perfusion. This study evaluates the use of gating the thallium image to the ECG to assess both right and left ventricular function and perfusion. Gated thallium scans were obtained from 100 consecutive male patients undergoing coronary angiography. Eighty megabecquerels of thallium 201 were injected intravenously during the last 30 seconds of maximal exercise and scans obtained in the anterior, $40^{\circ}$ and $70^{\circ}$ left anterior oblique projections, each for $5 \mathrm{~min}$ using a gammacamera. Analysis was performed by three independent observers, normal values being obtained from 12 healthy volunteers. Regional wall motion was assessed visually from the gated image and interobserver variation was small ( $0.9 \%$ for 1280 segments). Coronary angiography was performed within 48 hours of scanning, with a $50 \%$ reduction in luminal diameter being considered significant. Segmental perfusion defects were detected in $89 \%$ of patients with significant coronary disease, $75 \%$ with defects subtended by arteries with $50 \%$ $75 \%$ stenosis, and $94 \%$ with $99-100 \%$ stenosis. The addition of regional wall motion abnormalities identified $95 \%$ of patients with coronary disease. Fifty seven patients were noted to have inferior left ventricular defects, and right ventricular perfusion defects were seen in 20 of these scans. Seventy seven per cent of these patients had $>90 \%$ obstruction of the right coronary artery.

In conclusion, coronary artery lesions greater than $50 \%$ produce exercise perfusion defects and wall motion abnormalities of both the right and left ventricle, which are reliably detected by gated thallium scanning.

T lymphocyte subsets and function in dilated cardiomyopathy in East Africa

J E Sanderson, D Koech, D Iha, H P Ojiambo St Mary's Hospital, London, and the University of Nairobi Medical School, Kenya

It has been suggested that an excessive or inappropriate immune reaction may be partly responsible for the myocardial damage which leads to dilated car- diomyopathy. In order to determine if there is such a reaction in patients with dilated cardiomyopathy (DC) in East Africa we have measured the percentage of $T$ lymphocyte subsets using monoclonal antibodies (Ortho) to identify the total percentage of $\mathrm{T}$ lymphocytes (OKT3 positive), helper-inducer T cells (OKT4 positive), and suppressor-cytotoxic $\mathrm{T}$ cells (OKT8 positive) in 20 patients (nine men; mean age $32.8(13)$ years) with DC and 20 age and sex matched normal controls. The results showed that the total percentage of $\mathrm{T}$ cells were similar in both groups (patients: 65.6(2.6)\%; controls: $64 \cdot 1(2.0) \%$ ) while the percentage of helper-inducer cells was significantly higher in the DC group (patients: $44.7(1.8) \%$; controls: $32.8(2.0) \% ; \mathrm{p}<0.001)$ and the percentage of suppressor cells was slightly lower (patients: $28 \cdot 4(2 \cdot 2) \%$; controls: $33 \cdot 6(1 \cdot 6) \%)$. The helper:suppressor cell ratio was higher than the normal range in eight of the 20 patients. Seven of the eight patients with these high ratios were studied within three months of onset of their symptoms. Repeat measurements were done in seven patients, and in one patient there was a fall in the helper:suppressor ratio over five months. Lymphocyte functional assays (mixed lymphocyte reaction) were done in six patients and six controls, and in three patients the "stimulation index" was above the control group range. These results indicate that an excessive immune reaction may be part of the pathogenesis of dilated cardiomyopathy in Africa.

\section{Cardiac hypertrophy, hypertrophic cardiomyopathy and hyperparathyroidism - an association}

Farieda Fortune, R A Greenbaum, P Dandona, C Symons

The Royal Free Hospital, London

Patients with primary hyperparathyroidism were investigated to detect and categorise cardiac hypertrophy. In a separate group with hypertrophic cardiomyopathy, estimation of parathyroid hormone level was made. A third group with hypercalcaemia alone was studied. All had M mode echocardiography. Estimation of septal and left ventricular wall thickness was made at mitral valve closure, and minimum cavity dimension was estimated. The presence of systolic anterior motion of the mitral valve and mid-systolic closure of the aortic valve were noted. Echocardiographic estimations were made without the knowledge of the patient's biochemical status. Echocardiograms were classified as normal, symmetric left ventricular hypertrophy, asymmetric septal hypertrophy, and hypertrophic cardiomyopathy. 
Parathyroid hormone was measured by radioimmunoassay using an antibody technique directed against the mid-molecular peptide of the hormone. Forty one patients were studied: namely, $(a)$ primary hyperparathyroidism (16 cases): five (31\%) had symmetric left ventricular hypertrophy, five had hypertrophic cardiomyopathy, four had asymmetric septal hypertrophy, and two had no ventricular hypertrophy. All were hypercalcaemic. (b) Hypertrophic cardiomyopathy (18 cases): five (28\%) were found to have high levels of circulating parathyroid hormone. None had hypercalcaemia. (c) Hypercalcaemia without hyperparathyroidism (seven cases): none had any cardiac hypertrophy.

It is concluded that $(a)$ excess circulating parathyroid hormone is frequently associated with cardiac hypertrophy, including hypertrophic cardiomyopathy and $(b)$ hypercalcaemia of itself does not give rise to cardiac hypertrophy.

\section{Persistent echocardiographic and endomyocardial biopsy abnormalities after Coxsackie B myopericarditis}

D O'Neill, G Lindop, H J Dargie

Departments of Cardiology and Pathology, Western Infirmary, Glasgow

In order to assess the long term effects on cardiac function of Coxsackie B myopericarditis (CM) we performed $M$ mode echocardiography on 47 consecutive patients (24 male and 23 female, aged 42(13) years at a mean follow up time of 40 months (range 6-70 months) after diagnosis. These patients were compared with 42 normal control subjects ( 20 male and 22 female, aged 32(12) years. Mean right ventricular dimension was significantly greater at $2.35 \mathrm{~cm}$ in the CM group compared with $1.95 \mathrm{~cm}$ in the control group $(p<0.001)$; increased right ventricular dimension $(>2.6 \mathrm{~cm})$ was present in 11 patients, and in seven this was the only abnormality found. Mean left ventricular internal dimensions were similar in both groups but in three patients left ventricular end diastolic dimensions were similar in both groups but in three patients left ventricular end diastolic dimension was increased $(>5.7 \mathrm{~cm})$, and seven patients showed a reduction in left ventricular shortening fraction to $<25 \%$. In all, echocardiographic abnormalities were found in 16 patients (34\%), of whom 13 were male. Nine patients, eight of whom had an abnormal echocardiogram, also underwent endomyocardial biopsy. Three biopsies showed continuing active myocarditis with ongoing myocyte destruction, and six showed focal inflammation with replacement fibrosis indicating healing myocarditis. We conclude that persisting echocardiographic abnormalities are common after $\mathrm{CM}$, right ventricular enlargement being the most frequent finding which may occur in the absence of detectable left ventricular abnormality. Persistent low grade myocarditis may occur even in the absence of symptoms or cardiac enlargement. No progression to dilated cardiomyopathy has been observed so far.

Right ventricular wall thickness in hypertrophic cardiomyopathy: the relationship to clinical and prognostic features

\author{
R Foale, A Kleinebenne, P Nihoyannopoulos, \\ W J McKenna \\ Hammersmith Hospital, Royal Postgraduate Medical \\ School, London
}

Right ventricular hypertrophy (RVH) is found at autopsy in the majority of patients with hypertrophic cardiomyopathy (HCM) who die suddenly. Until recently it has been difficult to measure right ventricular wall thickness in vivo. We have performed dual $M$ mode and cross sectional echocardiography using right ventricular specific views to assess the presence of RVH and its relationship to prognostic features in 83 consecutive adults with HCM. Twenty four normal subjects were studied for comparison. Anterior, posterior, and diaphragmatic right ventricular wall thickness was evaluated from 13 right ventricular segments; 73 (88\%) of patients with HCM and 20 $(83 \%)$ normal subjects had recordings from which at least eight segments could be measured. In patients with $\mathrm{HCM}$, right ventricular wall thickness ranged from 3-20 $\mathrm{mm}$ compared to the normal group in which right ventricular wall thickness measured 4 mm. Thirty two (44\%) patients with HCM had at least one segment $>5 \mathrm{~mm}$ and were thus considered to have RVH. In this group, the coefficient of variation of right ventricular wall thickness was 0-30\% (median 9). In HCM patients without RVH, the coefficient of variation was $0-15 \%$ (median 11), which was similar to normals, 0-15\% (median 10). RVH in patients with HCM was symmetric in all but three and was more common in those with severe left ventricular hypertrophy (LVH of $20 \mathrm{~mm}(\mathrm{p}<0.02)$. RVH was neither related nor associated with a raised LVEDP, pulmonary arteriolar resistance, and RV or LV gradients. RVH was associated with dyspnoea $(p<0.05)$ and with ventricular tachycardia during 48 hour Holter ECG $(p<0.01)$. RVH is common in HCM, appears to 
be due to primary myopathic involvement rather than changes secondary to LV dysfunction, and is associated with ventricular tachycardia. RVH may improve the specificity of ventricular tachycardia as a marker for sudden death in adults with HCM.

\section{Prognostic indicators in dilated cardiomyopathy}

\author{
R Diaz, A Obasohan, H Newman, J F Goodwin, \\ Celia Oakley \\ Hammersmith Hospital, Royal Postgraduate Medical \\ School, London
}

One hundred and sixty nine patients aged 18 to 64 years with dilated cardiomyopathy were studied. Known causes of left ventricular failure were excluded by definition. All patients underwent cardiac catheterisation and none had coronary artery disease. At the time of diagnosis the left ventricular end diastolic pressure was elevated $(24(10) \mathrm{mm} \mathrm{Hg}$ ); the ejection fraction was depressed $(29(13) \%)$; and both the left ventricular end systolic volume $(105(57) \mathrm{ml} /$ $\mathrm{m}^{2}$ ) and the left ventricular end diastolic volume $\left(148(64) \mathrm{ml} / \mathrm{m}^{2}\right)$ were increased. The mean follow up was $5 \cdot 5(4 \cdot 2)$ years. During this period $102(60 \%)$ patients died. The average duration from the onset of symptoms to death was 4.1 (3.7) years. One year and five year mortality rates were $34.7 \%$ and $59.4 \%$ respectively with most deaths (44.1\%) occurring during the first two years of follow up. When the characteristics of the survivors and non-survivors were compared non-survivors had higher left ventricular end diastolic pressure (23 compared with $17 \mathrm{~mm} \mathrm{Hg}$; $\mathbf{p}<0.001$ ); lower ejection fraction ( 25 compared with $33 \% ; \mathrm{p}<0.005)$; and larger left ventricular end systolic volume (129 compared with $87 \mathrm{ml} / \mathrm{m}^{2} ; \mathrm{p}<0.01$ ) and left ventricular end diastolic volume (173 compared with $\left.131 \mathrm{ml} / \mathrm{m}^{2} ; \mathrm{p}<0.01\right)$ than did the survivors. Recent pregnancy and heavy alcohol intake did not seem to influence prognosis. Ventricular tachycardia or multiforms on Holter recording were more common in the non-survivors but were a feature of worse left ventricles and not an independent risk factor. When treatment was compared no differences were found between the two groups and vasodilators could not be shown to influence survival, having been given to about a quarter of the patients in each group. Dilated cardiomyopathy has a high mortality with survival time apparently related only to the severity of left ventricular impairment at presentation.
Radiation hazards associated with percutaneous transluminal coronary angioplasty

K Faulkener, H G Love, R Bardsley, S T Jeans,
J Sweeney
Christie Hospital and Holt Radium Institute and
Manchester Royal Infirmary, Manchester

Percutaneous transluminal coronary angioplasty is a recognised technique in the management of coronary disease. The Simpson method as been adopted in this unit. The radiation dosage to patients and operators was studied. Scattered dose measurements were made at the thyroid, ovaries, and testes in 15 patients. Whole body integral doses to patients were determined from exposure area products. Doses to patients decreased with experience and new guide wire systems. Data concerning staff indicated the operator would exceed the occupational dose limit to the eye if more than 120 percutaneous transluminal coronary angioplasties were performed per year. When other invasive procedures including catheterisation and pacing were included this limit dropped to only 60 examinations per year. Likewise repeat cardiac catheterisation increases gonadal doses to patients.

The following recommendations are made $(a)$ operators should consider extra eye protection such as lead glass spectacles, photochromic lenses, or portable acrylic screens and $(b)$ patients undergoing percutaneous transluminal coronary angioplasty during the reproductive period should have gonadal protection at the time of angioplasty and during subsequent procedures. It is emphasised that the operator is at major risk of cataract development if such precautions are not taken.

Pericardoscopy-a new approach in the management of pericardial effusions of unknown aetiology

\section{S Westaby}

Royal Postgraduate Medical School, Hammersmith Hospital, London

Treatment of reaccumulating pericardial effusions with cardiac tamponade often presents difficulties. Failure to obtain a diagnosis by needle aspiration and cytology is usually followed by subxiphoid surgical drainage, though access to anything but the diaphragmatic pericardium is limited. Pericardoscopy was used to inspect the interior of the pericardium in 
seven patients with large effusions of unknown aetiology. Under general anaesthesia the pericardium was approached by the subxiphoid route and drained through a small incision. The sac was then washed with warm saline and refilled. Visual inspection was carried out with both a cystoscope and fibreoptic bronchoscope. The anterolateral aspects of the heart and pericardium were easily seen with the rigid instrument. The fibreoptic scope could be passed around the heart to the posterior pericardium. In five patients otherwise inaccessible invasive tumours were seen and biopsied. Two were bronchogenic, one thymic, one a mesothelioma, and one adenocarcinoma. Two patients had infective effusions, one tuberculous and the other viral. Pericardectomy was carried out under the same anaesthetic in the tuberculous patient. The only adverse effects were ectopic beats when the heart was touched and minor bleeding from the biopsy sites. A pericardial drain was left in situ at the end of the procedure in each case.

In conclusion pericardoscopy provides useful information in patients with pericardial effusion of unknown aetiology who require surgical pericardial drainage.

\section{Left ventricular bands: a morphological study}

\author{
L M Gerlis, Helena Wright, N Wilson, D F \\ Dickinson \\ Killingbeck Hospital, Leeds
}

Discrete fibromuscular structures crossing the left ventricular cavity (LV bands) were recognised many years ago but recently cross sections echocardiography has resulted in a renewal of interest. It has been suggested that they might be responsible for a variety of problems. To assess their significance we studied autopsy specimens from 636 children's hearts ( 581 with congenital malformation, 55 normal) and 50 adult hearts with acquired disease. We also studied 159 hearts from six different animal species. LV bands were present in $47.7 \%$ of the children's hearts (with no difference in prevalence between the normal hearts and those with congenital malformations) and in $52 \%$ of the adult hearts. In $20 \%$ of cases with bands the bands were multiple. Almost invariably bands were attached to the septum at one end with the peripheral attachment to either papillary muscle or the ventricular free wall. In animals, bands were present in $100 \%$ of sheep, cats, horses, and dogs, in $86 \%$ of pigs, and $92 \%$ of oxen. A characteristic pattern was present in each species. Left ventricular bands are common and might therefore by chance alone be associated with almost any physical sign or symptom.

Increased prevalence of mitral valve prolapse associated with an elevated type III:III + I collagen ratio

C E Handler, A H Child

Guy's and St George's Hospitals, London

The precise biochemical basis for the mitral valve prolapse syndrome remains unclear. We have assessed the presence of mitral valve prolapse clinically and with phono and $M$ mode and cross sectional echocardiography in 14 female patients (age range 22-57 years) who had joint hypermobility. A raised type III:III + I collagen ratio (range $24-46 \%$, mean $30.7 \%$ ) (normal $21-23 \%$ ), measured by a new method using sodium dodecylsulphate polyacrylamide electrophoresis from skin biopsies, was found in all the eight patients samples. An increased aortic wall compliance (100-217\%, mean 150.7\%) (normal $80-120 \%$ ), measured by Doppler ultrasound, was found in 13 patients. Nine patients $(64 \%)$ had mitral valve prolapse, a prevalence at least three times greater than that in the adult population. There was also a trend for an increased prevalence of mitral valve prolapse and higher collagen III:III + I ratios with increasing age.

We conclude that although mitral valve prolapse was not a totally consistent finding an abnormality of collagen biosynthesis may be an important cause of this common cardiac condition. 